\title{
Uma análise da difração de elétrons por um fio delgado de comprimento variado com e sem efeitos de fase Aharonov-Bohm
}

An analysis of the diffraction of electrons by a thin wire of varied length with and without Aharonov-Bohm phase effects

\author{
Denise Assafrão ${ }^{1}$, Cássio C. Favarato ${ }^{1}$, Sergio V. B. Gonçalves*1] \\ ${ }^{1}$ Universidade Federal do Espírito Santo, Centro de Ciências Exatas, Departamento de Física, 29075-910, Vitória, ES, Brasil
}

Recebido em 06 de Agosto, 2017. Aceito em 13 de Setembro, 2018.

\begin{abstract}
É comum, durante os cursos de graduação, estudarmos o fenômeno da difração de ondas eletromagnéticas por sistemas de fendas simples e dupla via teoria escalar da difração e a integral de Kirchoff. De maneira alternativa, analisamos aqui nesse trabalho os padrões de difração de elétrons por uma barreira ou fio delgado de comprimento variado, com e sem efeito de fase Aharonov-Bohm, via integrais de caminho de Feynman. Neste contexto observamos o gradativo surgimento e a predominância dos diferentes domínios da óptica, a física e a geométrica, na região imediatamente anterior ao obstáculo, nos levando a um comportamento complementar ao observado na difração por fenda simples, em particular, quando o obstáculo torna-se muito maior que o comprimento de onda $\lambda$.

Palavras-chave: Difração, Funções de Fresnel, Integrais de caminho, Efeito Aharonov-Bohm.
\end{abstract}

\begin{abstract}
It is common during undergraduate courses to study the phenomenon of diffraction of electromagnetic waves by single and double slit systems via scalar diffraction theory and the Kirchoff integral. Alternatively, we analyze here the diffraction patterns of electrons by a barrier or thin wire of varied length, with and without Aharonov-Bohm phase effect, via Feynman path integrals. In this context we observe the gradual emergence and predominance of the different domains of optics, the physical and the geometric, in the region immediately before the obstacle, leading to a behavior complementary to that observed in simple slit diffraction, in particular, when the obstacle becomes much larger than the wavelength $\lambda$
\end{abstract}

Keywords: Aharonov-Bohm effect, path integrals, Fresnel functions, Difraction.

\section{Introdução}

Após o trabalho de L. de Broglie [1] sobre o princípio da dualidade onda-partícula, Davisson e Germer [2], confirmaram a natureza dual do elétron por vias experimentais. O problema quântico de difração e interferência de partículas massivas também foi discutido por R.P. Feynman 3 4. Décadas mais tarde, Roger Bach et al. 5] demonstraram a plena realização experimental do mecanismo idealizado por Feynman em 3]. Outra observação experimental pioneira foi feita por Jonson em 1961 [6] estendendo a análise da difração por múltiplas fendas em 1974 [7. É na década de 70 do século passado que ocorre a primeira prova experimental da difração quântica de elétrons individuais [8, 9 , que mais tarde, e de forma independente, também foi confirmado por outros trabalhos importantes [5.10. Já em 2012, Rodolfo Rosa [11] examinou o experimento proposto por P. G. Merli et al. em [9], colocando-o em um contexto histórico e discutindo suas implicações filosóficas. Com os artifícios modernos e práticos que a nanotecnologia proporciona 12], S.Fribboni et al. 13] construíram fendas em escala submicrométricas de modo que as experiências de interferência e difração de elétrons pudessem ser feitas mesmo com um microscópio eletrônico convencional. Somado a isso, experimentos de fenda foram realizados com nêutrons 14], átomos ultrafrios 15], átomos de Hélio 16 e com moléculas pesadas, como o $C_{60}$ e o $C_{70}[17 \sqrt{19}$. M. Gondran e A. Gondram [20] apresentaram uma simulação numérica do experimento abordado em [15] e usando o método das integrais de caminho de Feynman calcularam a função de onda dependente do tempo e as trajetórias na interpretação de Broglie-Bohm.

Em 21], Xiang-Yao Wu et al. examinam a difração de neutrons por uma e duas fendas com particular atenção para nêutrons frios. Aplicando a representação de Schrödinger em consonância com o teorema integral de

*Endereço de correspondência: sergiovbg@gmail.com. 
Kirchhoff, eles encontraram duas expressões para a intensidade observada. A primeira expressão decorre de um estado de superposição coerente, isto é, uma simples combinação linear das funções de onda emergentes de cada uma das fendas, desconsiderando assim quaisquer interações entre o sistema e o meio onde está inserido. A segunda expressão surge também de uma combinação linear das funções procedentes de cada fenda, porém são acompanhadas por estados que denotavam a influência do meio exterior. Os autores puderam então constatar que ao considerarem a superposição coerente o resultado teórico apresentava certa discrepância com os dados experimentais apresentados em [14]. Essa diferença pode ser eliminada quando se considera o mecanismo de descoerência. A. S. Sanz e F. Borondo 22], também avaliaram os resultados de [14], com o objetivo de obter uma melhor conformidade entre a teoria e o que foi medido.

Em trabalho recente 23, seus autores afirmam que um feixe luminoso ao adentrar por uma fenda (cujas dimensões são comparáveis com a ordem de grandeza do comprimento de onda do feixe de partículas em questão) mostra no anteparo um alargamento desse feixe e sua penetração na região de sombra geométrica, acompanhado do surgimento de franjas de interferência claras e escuras nas proximidades do limite da sombra, típicas do fenômeno de difração. Esse fenômeno, que se estende para um feixe de elétrons (na referência 24], o leitor encontrará uma interessante discussão sobre a natureza do elétron, assim como em [25], onde são discutidos dois experimentos que lidam, didaticamente, com a natureza ondulatória da luz e da matéria), também se manifesta quando a onda incide sobre um obstáculo com dimensões comparáveis ao seu comprimento de onda. Nesse ponto temos a seguinte questão: o que ocorre quando a dimensão do objeto é muito maior, muito menor ou aproximadamente da mesma ordem de grandeza do comprimento de onda do feixe de partículas?

A proposta deste trabalho é encontrar, usando a representação das integrais de trajetória da Mecânica Quântica (MQ) 26, 27. os padrões de interferência gerados pela incidência de elétrons sobre um obstáculo delgado, com e sem efeito de fase Aharonov-Bohm (AB). Em 28], o autor demonstra que certos parâmetros físicos, em particular os números de Fresnel, estarão diretamente vinculados as formas gráficas dos padrões de interferência e difração, por fenda simples e dupla, o que nos leva a diferentes regimes ópticos. Ele apresenta analiticamente as expressões das intensidades dos elétrons sobre a tela de observação e a partir dela, faz algumas aproximações baseadas no comportamento assintótico das funções de Fresnel, proporcionando-nos assim um entendimento mais refinado das curvas de interferência dentro do espectro de cada um desses regimes. Parte dessas considerações também serão atribuídas ao nosso modelo, nos permitindo avaliar de maneira simples e didática as curvas de interferência com base na relação direta entre o comprimento de onda do elétron e o tamanho característico do obstá- culo que, ao variar, denunciam os diferentes domínios da óptica, isto é, a física e a geométrica.

\section{O hipotético experimento de interferência e difração}

Vamos considerar nesse trabalho o modelo conceitual originalmente concebido em [4] e revisitado com maiores detalhes em 28 30]. Assim, vamos estudar o movimento em duas regiões: a primeira, partindo da fonte para as regiões que denominamos de semi fendas $( \pm)$, no intervalo de tempo $T$, e a segunda, partindo destas para a tela durante o intervalo de tempo $\tau$. A partir daí, vamos determinar a função de onda para um elétron, que sai da fonte em $(x=0, y=0, z=0)$ em $t=0$, e chega à tela, na posição $(x, z=D+L)$ no instante $t=T+\tau$, sabendo que o mesmo atravessa uma região intermediária em $(\omega, z=D)$, com $-\infty<\omega<-b$ e $+b<\omega<+\infty$ em $t=T$ (conforme pode ser visto na Fig. 1). A julgar pelas regras da $\mathrm{MQ}$, não haveria nenhuma razão para separar o movimento em duas partes independentes, uma vez que não é conhecida a posição da partícula no instante $T$. Isto é, pelo princípio da incerteza, não é possível afirmar com precisão absoluta o momento em que a partícula atravessa as semi fendas. No entanto, como em todas as demais referências 28 34, esta abordagem semi-clássica é razoavelmente apropriada ao estudo desse problema. Isso se justifica uma vez que, na direção $z$ o elétron tem como componente de momento linear $p_{z}=K_{z} \hbar$, com $K_{z}$ a componente do vetor de onda nesta direção, que está relacionada à componente da velocidade clássica $v_{z}=D / T=L / \tau$ com $D$ supostamente muito maior que as dimensões consideradas na direção $x$, isto é, $(x, b)$ $\ll(D, L)$. Além disso, é suposto que o comprimento de onda $\lambda$, que por sua vez é aproximadamente igual a sua componente na direção $z, \lambda \simeq \lambda_{z}=2 \pi \hbar /\left(m v_{z}\right)$, é muito menor que as distâncias $D$ e $L(\lambda \ll(D, L))$. Dessa forma, na direção $z$, o movimento é aproximadamente clássico.

Formulado por R. Feynman 35 em 1948, o conceito de integral de caminho baseia-se em um artigo de A. P. M. Dirac 36. Sua proposta fundamentava-se no princípio de mínima ação quântica: "A amplitude de transição sobre os estados $|a\rangle$ e $|b\rangle$ de um sistema quântico-mecânico é a soma das contribuições elementares, uma para cada trajetória, passando em $|a\rangle$ no instante $t_{a}$, e em $|b\rangle$ no instante $t_{b}$. Cada uma dessas contribuições possui o mesmo módulo, porém sua fase é a ação clássica $\mathcal{S}_{c l}$ para cada caminho" 4]. Esse princípio é traduzido pela seguinte expressão:

$$
\mathcal{K}(a \rightarrow b)=\int_{a}^{b} \exp \left(\frac{i}{\hbar} \mathcal{S}_{c l}\right) \mathrm{D}[x(t)],
$$

em que

$$
\mathcal{S}_{c l}(b, a)=\int_{a}^{b} \mathcal{L}(q, \dot{q}, t) d t
$$


e $D[x(t)]$ é conhecido como a medida de Feynman 37 . A integral que define $\mathcal{K}(a \rightarrow b)$ é chamada de Integral de caminho ou propagador de Feynman 44.

Considere $K_{0}$ o propagador livre associado a dinâmica dos elétrons na ausência de quaisquer interações, em cada subintervalo do movimento. Com base na Fig. 1, o propagador da fonte a cada uma das semi fendas $( \pm)$ é dada por,

$$
\begin{aligned}
\mathcal{K}_{0}\left(F^{( \pm)}, \mathcal{O}\right) \longrightarrow \mathcal{K}_{0}(\omega, T ; 0,0) & =\left(\frac{m}{2 \pi i \hbar\left(t_{F^{( \pm)}}-t_{\mathcal{O}}\right)}\right)^{1 / 2} \exp \left(\frac{i m}{2 \hbar} \frac{\left|F^{( \pm)}-\mathcal{O}\right|^{2}}{\left(t_{F^{( \pm)}}-t_{\mathcal{O}}\right)}\right) \\
& =\left(\frac{m}{2 \pi i \hbar T}\right)^{1 / 2} \exp \left(\frac{i m}{2 \hbar} \frac{\left(\omega^{2}+D^{2}\right)}{T}\right)
\end{aligned}
$$

Os propagadores do plano das semi fendas a tela serão dados por

$$
\begin{aligned}
\mathcal{K}_{0}\left(\mathcal{X}^{\prime( \pm)}, F^{( \pm)}\right) & \longrightarrow \mathcal{K}_{0}(x, T+\tau ; \omega, T) \\
& =\left(\frac{m}{2 \pi i \hbar\left(t_{\mathcal{X}^{\prime}( \pm)}-t_{F^{( \pm)}}\right)}\right)^{1 / 2} \exp \left(\frac{i m}{2 \hbar} \frac{\left|\mathcal{X}^{\prime( \pm)}-F^{( \pm)}\right|^{2}}{\left(t_{\mathcal{X}^{( \pm)}}-t_{F^{( \pm)}}\right)}\right) \\
& =\left(\frac{m}{2 \pi i \hbar \tau}\right)^{1 / 2} \exp \left(\frac{i m}{2 \hbar} \frac{\left(L^{2}+\left( \pm x^{2} \mp \omega^{2}\right)\right)}{\tau}\right)
\end{aligned}
$$

Em 1979, D. H. Kobe 29], argumenta que a representação por integrais de caminho proporciona uma maneira muito natural de lidar com a difração, já que a amplitude de probabilidade total de um elétron propagar-se de um ponto ao outro no espaço-tempo é dado em termos de uma soma de amplitudes, uma para cada caminho individual, com caminhos proibidos devido as barreiras sendo facilmente excluídos dessa soma. Podemos representar o obstáculo e as regiões de semi fendas $( \pm)$ através da função,

$$
\aleph_{[(-),(+)]}(\omega)= \begin{cases}0 & \text { se }-b \leq \omega \leq 0, \quad 0 \leq \omega \leq+b, \\ 1 & \text { se }-\infty<\omega<-b, \quad+b<\omega<+\infty .\end{cases}
$$

As funções de onda emergentes de cada uma das semi fendas $( \pm)$ sã escritas como,

$$
\Psi^{( \pm)}(x)=\int_{-\infty}^{\infty} \mathcal{K}_{0}\left(\mathcal{X}^{( \pm)}, F^{( \pm)}\right) \aleph_{[(-),(+)]}(\omega) \mathcal{K}_{0}\left(F^{( \pm)}, \mathcal{O}\right) d \omega
$$

Nota-se que a função $\aleph_{[-b,+b]}(\omega)$ é responsável por fixar os limites de cada integral, limitando as possíveis trajetórias ao longo do eixo do obstáculo. Podemos escrever as funções de onda emergentes das regiões abertas da seguinte

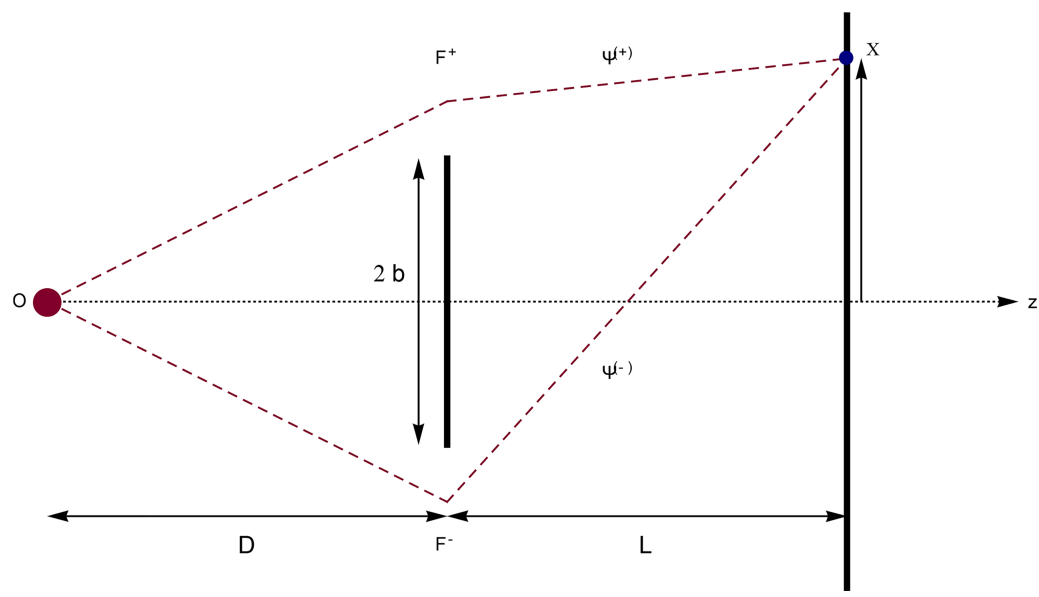

Figura 1: Representação gráfica do aparato com a fonte e os respectivos eixos, do obstáculo (um fio delgado) e da tela de observação. Os segmentos tracejados denotam os chamados caminhos clássicos, isto é, aqueles que extremizam a ação, Eq. (2), assumidos pelas partículas, sendo $\Psi^{(+)}$e $\Psi^{(-)}$as funções de onda emergentes de cada uma dessas regiões. 
maneira

$$
\begin{aligned}
\Psi^{( \pm)}(x) & =\sqrt{\frac{2}{\pi} \nu} \mathcal{N} e^{i(\xi+\vartheta)}\left\{\left[\mathcal{C}\left(\zeta_{2}^{( \pm)}\right)+i \mathcal{S}\left(\zeta_{2}^{( \pm)}\right)\right]-\left[\mathcal{C}\left(\zeta_{1}^{( \pm)}\right)+i \mathcal{S}\left(\zeta_{1}^{( \pm)}\right)\right]\right\} \\
& =\sqrt{\frac{2}{\pi} \nu} \mathcal{N} e^{i(\xi+\vartheta)}\left\{\left[\mathcal{C}\left(\zeta_{2}^{( \pm)}\right)-\mathcal{C}\left(\zeta_{1}^{( \pm)}\right)\right]+i\left[\mathcal{S}\left(\zeta_{2}^{( \pm)}\right)-\mathcal{S}\left(\zeta_{1}^{( \pm)}\right)\right]\right\} \\
& =\sqrt{\frac{2}{\pi}} \nu \mathcal{N} e^{i(\xi+\vartheta)}\left[\Delta \mathcal{C}\left(\zeta^{( \pm)}\right)+i \Delta \mathcal{S}\left(\zeta^{( \pm)}\right)\right]
\end{aligned}
$$

com os fatores multiplicativos e os termos nas exponenciais dados por

$$
\begin{aligned}
& \mathcal{N}=\left(\frac{m}{2 \pi i \hbar \sqrt{T \tau}}\right), \nu=\frac{m}{2 \hbar}\left(\frac{1}{T}+\frac{1}{\tau}\right) \\
& \xi=\exp \left[\frac{m}{2 \hbar}\left(\frac{D^{2}}{T}+\frac{L^{2}}{\tau}\right)\right], \vartheta^{( \pm)}=\frac{m x^{2}}{2 \hbar(T+\tau)}(8)
\end{aligned}
$$

Integrais de funções trigonométricas com argumentos que são polinômios de grau dois, presentes na eq. (7) são denominadas de integrais de Fresnel dessas respectivas funções, sendo representadas por

$$
\begin{aligned}
& \mathcal{C}\left(\zeta_{(2 / 1)}^{( \pm)}\right)=\int_{0}^{\zeta_{(2 / 1)}^{( \pm)}} \cos \left(\frac{\pi}{2}\left(\zeta^{( \pm)}\right)^{2}\right) d \zeta^{( \pm)} \\
& \mathcal{S}\left(\zeta_{(2 / 1)}^{( \pm)}\right)=\int_{0}^{\zeta_{(2 / 1)}^{( \pm)}} \sin \left(\frac{\pi}{2}\left(\zeta^{( \pm)}\right)^{2}\right) d \zeta^{( \pm)} .
\end{aligned}
$$

Ambas são integrais resolvidas numericamente, conforme 38 40]. Da Fig. 2 percebemos que essas funções são ím- pares de seus argumentos, convergindo para os seguintes valores quando tomados os limites

$$
\begin{aligned}
\lim _{\zeta \rightarrow \pm \infty} \mathcal{C}(\zeta) & = \pm 1 / 2, \\
\lim _{\zeta \rightarrow \pm \infty} \mathcal{S}(\zeta) & = \pm 1 / 2 .
\end{aligned}
$$

Essas funções eram calculadas através da espiral de Cornu [4], uma curva gerada por uma representação paramétrica de $\mathcal{S}(\zeta)$ e $\mathcal{C}(\zeta)$ no plano complexo, como pode ser visto na Fig. 3.

Também conhecida como radióide de arcos, clotóide ou espiral de Euler, essa curva foi descoberta por Euler em 1744 e utilizada mais tarde por M. A. Cornu como auxiliar no estudo da teoria da difração de Fresnel, com seu conceito usualmente aplicado na elaboração do desenho geométrico de rodovias e ferrovias [41 43]. Luís Blanch et al. 44 fizeram um estudo pormenorizado e muito didático sobre essa curva. Partindo das propriedades geométricas que definem uma Clotóide, eles obtiveram sua equação a partir das integrais de Fresnel (definidas

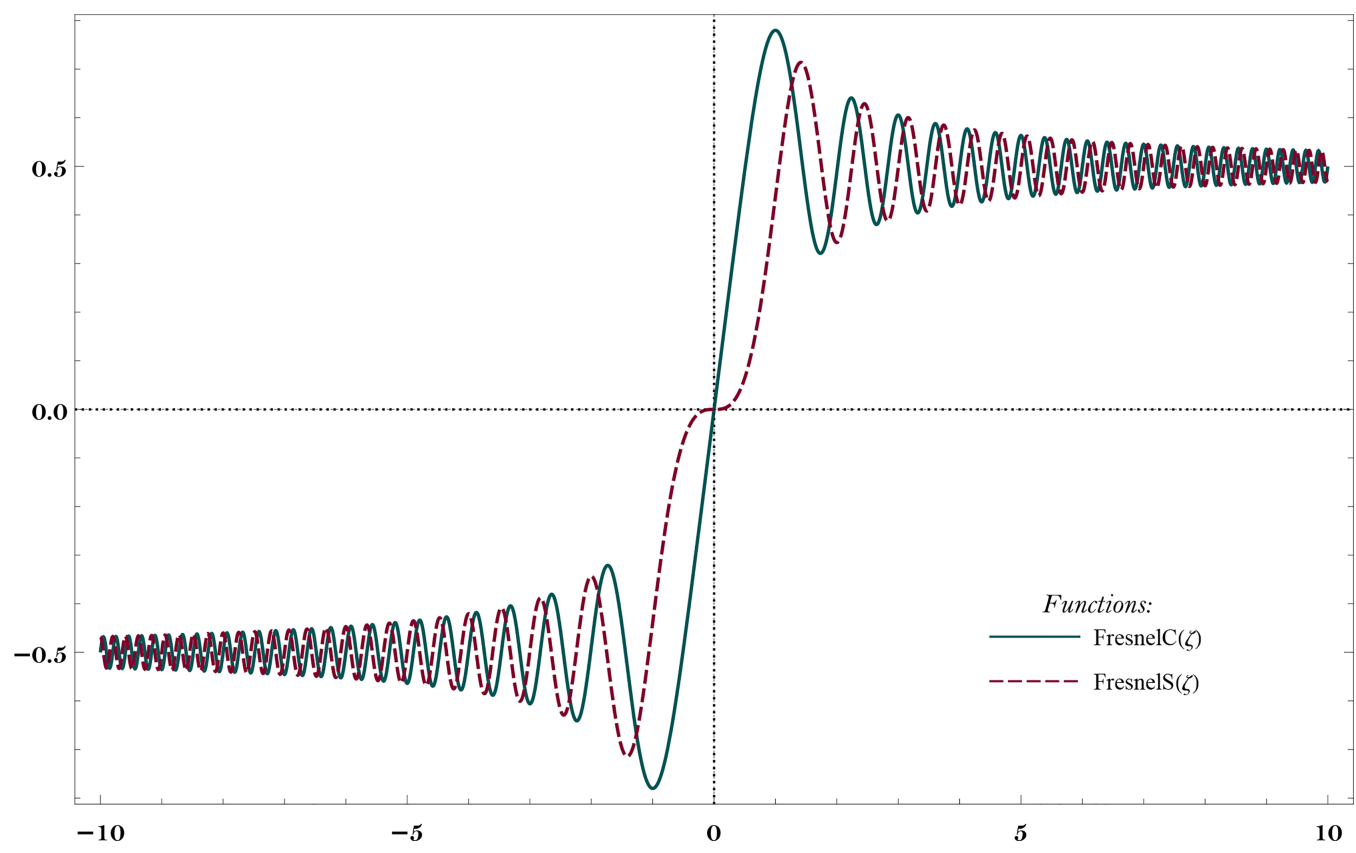

Figura 2: Comportamento das funções de Fresnel. 


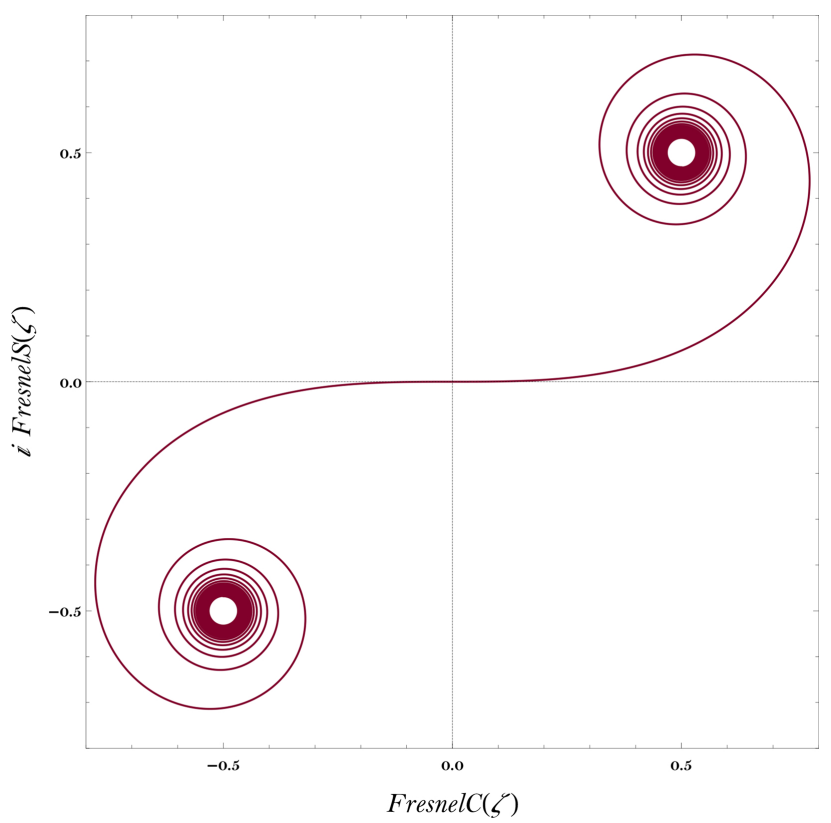

Figura 3: A representação paramétrica das funções de Fresnel no plano complexo são as espirais de Cornu.

em (9a) e (9b), representando geometricamente certas características encontradas e alcançando uma aproximação numérica para a mesma. Em 45, 46 podemos encontrar trabalhos que lidam com as funções de Fresnel em suas mais diversas formas e aplicações em problemas de espalhamento em Física Óptica.

De acordo com o princípio de Huygens, quando a onda incidente chega ao eixo obstáculo, todos os pontos pertencentes as regiões abertas $( \pm)$ tornam-se fontes de ondas secundárias (incluindo os pontos que fazem parte dos extremos do obstáculo), emissoras de novas ondas coerentes, cuja amplitude resultante é dada pela Eq. (7), quando consideradas cada uma delas isoladamente, como mostra a Fig. 4

As ondas provenientes desses pontos, ao se propagarem, combinam-se entre si gerando um padrão de interferência sobre a tela cuja forma dependerá da relação entre as dimensões do obstáculo $2 b$ e do comprimento de onda $\lambda$ do feixe (ver Fig. 1). Esta relação se traduz matematicamente através do chamado número de Fresnel,

$$
N_{F}(b) \equiv \frac{2 b^{2}}{\lambda L}
$$

O obstáculo possui superfície perfeitamente polida, não havendo qualquer tipo de interação e, portanto, os elétrons ao colidirem sobre ele, sofrem um espalhamento elástico preservando seu comprimento de onda. O módulo ao quadrado das funções $\Psi^{( \pm)}(x)$ na Eq. (7), nos fornecem as intensidades de difração para cada semi fenda,

$$
\begin{aligned}
\mathcal{P}^{( \pm)}(x) & =\Psi^{( \pm)^{*}}(x) \Psi^{( \pm)}(x) \\
& =\frac{1}{2 \lambda(D+L)}\left[\left(\mathcal{C}^{2}\left(\zeta_{\left\{1^{(+)} ; 2^{(-)}\right\}}\right)+\mathcal{S}^{2}\left(\zeta_{\left\{1^{(+)} ; 2^{(-)}\right\}}\right)\right)\right. \\
& \left.\mp \mathcal{C}\left(\zeta_{\left\{1^{(+)} ; 2^{(-)}\right\}}\right) \mp \mathcal{S}\left(\zeta_{\left\{1^{(+)} ; 2^{(-)}\right\}}\right)+\frac{1}{2}\right]
\end{aligned}
$$

Para fins práticos, ainda não discriminamos as formas dos argumentos das funções de Fresnel presentes nas Eq. (7) e Eq. 12), pois exploramos configurações geométricas distintas que auxiliarão no entendimento do problema.
$\mathrm{Na}$ primeira delas, idealizamos um aparato com uma única fenda centrada em $x=0$ e extremamente grande, ao ponto de nos permitir aplicar os limites dados em (10a) e 10b), convertendo a Eq. 12 em,

$$
\begin{aligned}
\mathcal{P}_{0} & =\frac{1}{2 \lambda(D+L)} \lim _{\zeta \rightarrow \infty}\left[(\mathcal{C}(\zeta)-\mathcal{C}(-\zeta))^{2}+(\mathcal{S}(\zeta)-\mathcal{S}(-\zeta))^{2}\right] \\
& =\frac{1}{2 \lambda(D+L)}\left[(1 / 2-(-1 / 2))^{2}+(1 / 2-(-1 / 2))^{2}\right], \\
& =\frac{1}{\lambda(D+L)}
\end{aligned}
$$

A partir disso, examinamos o caso particular descrito geometricamente por uma borda reta (semi fenda), cuja parede se estende de $(-\infty, 0]$ e abertura que vai de $(0,+\infty)$.
Isso implica adotar os limites,

$$
\begin{aligned}
\zeta_{2}^{(+)} & \rightarrow+\infty \\
\zeta_{1}^{(+)} \rightarrow \zeta^{(+)}(x ; \eta) & =-\sqrt{\frac{2}{\lambda L}} \frac{x}{\eta}
\end{aligned}
$$




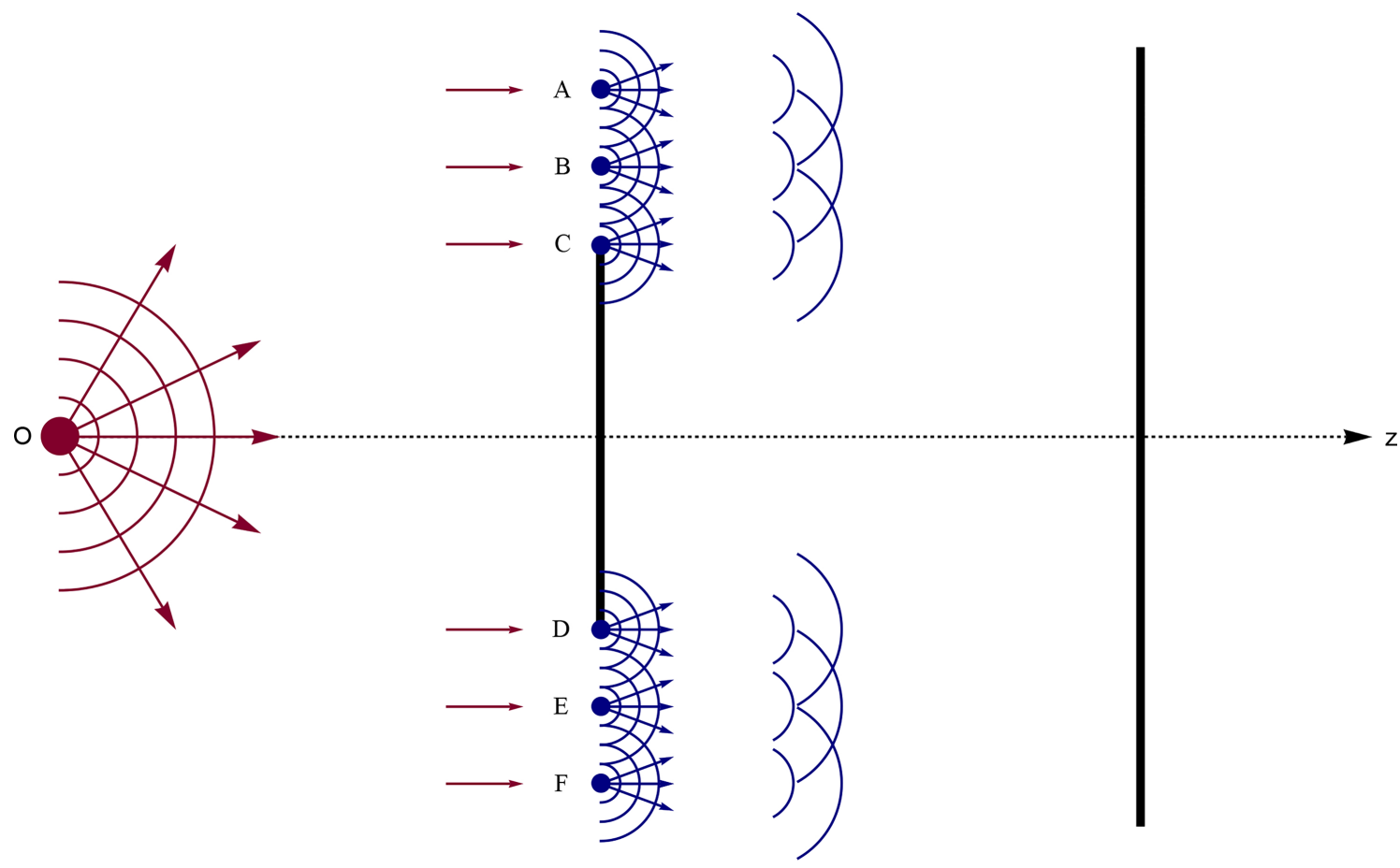

Figura 4: Representação gráfica do aparato com a fonte emissora de ondas esféricas (seções circulares no plano $x O z$ ). Sobre o eixo do obstáculo, nas regiões de semi fendas, são representados alguns pontos que pelo princípio de Huygens, agem como fontes secundárias.

considerando os parâmetros,

$$
\eta \equiv 1+\frac{L}{D} \quad \rightarrow \quad \rho=\eta-1
$$

Empregando 14a e (14b como argumentos das funções de Fresnel na Eq. 12$)\left(\right.$ para $\left.\mathcal{P}^{(+)}(x)\right)$, podemos avaliar analítica e graficamente o comportamento da intensidade,

$$
\begin{aligned}
\mathcal{P}^{(+)}(x) & =\frac{\mathcal{P}_{0}}{2}\left\{\left[1 / 2-\mathcal{C}\left(-\sqrt{\frac{2}{\lambda L}} \frac{x}{\eta}\right)\right]^{2}+\left[1 / 2-\mathcal{S}\left(-\sqrt{\frac{2}{\lambda L}} \frac{x}{\eta}\right)\right]^{2}\right\}, \\
& =\frac{\mathcal{P}_{0}}{2}\left\{\left[1 / 2+\mathcal{C}\left(\sqrt{\frac{2}{\lambda L}} \frac{x}{\eta}\right)\right]^{2}+\left[1 / 2+\mathcal{S}\left(\sqrt{\frac{2}{\lambda L}} \frac{x}{\eta}\right)\right]^{2}\right\} .
\end{aligned}
$$

Esta equação nos chama a atenção para alguns pontos interessantes, em particular àqueles que, se a óptica geométrica fosse estritamente válida, integrariam a região de sombra, isto é, de intensidade nula. Vejamos o que ocorre no limite geométrico da abertura,

$$
\begin{aligned}
\mathcal{P}^{(+)}(x=0) & =\frac{\mathcal{P}_{0}}{2}\left\{[1 / 2+\mathcal{C}(0)]^{2}\right. \\
& \left.+[1 / 2+\mathcal{S}(0)]^{2}\right\}=\frac{\mathcal{P}_{0}}{4} .
\end{aligned}
$$

A medida que penetramos a região de sombra, com $x$ assumindo valores negativos, a intensidade cai gradualmente até se anular $(x \rightarrow-\infty)$. Em contrapartida, quando $x$ se torna positivo (região geométrica de iluminação), ela aumenta, assumindo um valor máximo em aproximadamente 1.37, para em seguida cair, flutuando em torno de um valor constante, e por isso, adquirindo um aspecto gráfico serrilhado visto na Fig. 5

$$
\lim _{x \rightarrow \infty} \mathcal{P}^{(+)}(x)=\mathcal{P}_{0} .
$$

Note que neste caso, o conceito usual de fenda finita e mensurável com respeito a $\lambda$ se perde e diferentemente do que pode vir a ocorrer nos casos de fenda simples e dupla cujos padrões de interferência dependem fortemente da relação entre as dimensões das fendas, distâncias entre elas e de $\lambda$, em diferentes regimes ópticos, sua forma se preserva. Isso fica claro ao variarmos o parâmetro adimensional $\eta$, a intensidade é redistribuida, ficando comprimida $(L \ll D)$ ou distendida $(L \gg D)$, e neste caso a região "iluminada"vai se estendendo ainda mais fundo para valores de $x$ negativo. Mesmo assim, seu aspecto serrilhado, característico do regime de Fresnel [4], se mantém. 


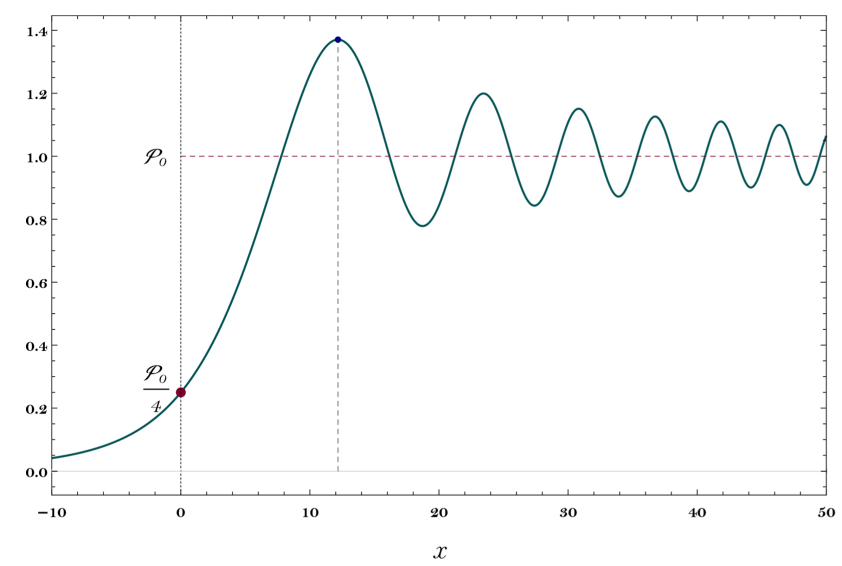

(a) $\quad \eta=20(L=19 D)$.

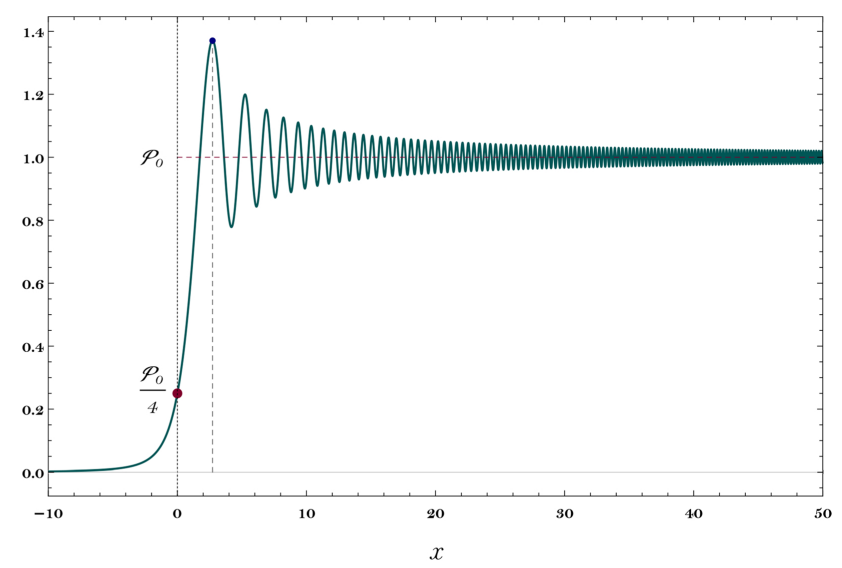

(b) $\quad \eta=1.001(L=0.001 D)$.

Figura 5: Padrão de difração por uma borda reta, mostrando as oscilações de intensidade em torno de $\mathcal{P}_{0}$, para $x$, graduado em unidades arbitrárias de comprimento, crescente.

Assim como no tradicional experimento de fenda dupla, a amplitude de transição ou função de onda total sobre a tela de detecção é, em geral, obtida pela simples adição das amplitudes correspondentes a cada uma das regiões de fenda (abertura). Logo, a função de onda total será, pelo princípio da superposição

$$
\begin{aligned}
& \Psi(x)=\Psi^{(+)}(x)+\Psi^{(-)}(x), \\
& =\sqrt{\frac{2}{\pi} \nu} \mathcal{N} e^{i(\xi+\vartheta)}\left[\left(\mathcal{C}\left(\zeta_{2}^{(-)}\right)-\mathcal{C}\left(\zeta_{1}^{(+)}\right)+1\right)\right. \\
& \left.+i\left(\mathcal{S}\left(\zeta_{2}^{(-)}\right)-\mathcal{S}\left(\zeta_{1}^{(+)}\right)+1\right)\right] \text {, }
\end{aligned}
$$

cujos argumentos das funções de Fresnel são, agora, dados por,

$$
\begin{aligned}
\zeta_{1}^{(+)} & =\sqrt{N_{F}(b) \eta}\left(1-\frac{x}{b \eta}\right) \\
\zeta_{2}^{(-)} & =\sqrt{N_{F}(b) \eta}\left(-1-\frac{x}{b \eta}\right) .
\end{aligned}
$$

Contudo, ressaltamos o fato que a Eq. (19) não descreve completamente o sistema. De acordo H.Yabiki em [48], tal como em seu experimento de fenda dupla, as funções de onda $\Psi(x), \Psi^{(+)}(x)$ e $\Psi^{(-)}(x)$, pertencentes a diferentes espaços de Hilbert, são tidas como soluções da equação de Schrödinger para diferentes condições de contorno. Desta forma, para corrigir este detalhe deveríamos levar em consideração a contribuição de caminhos exóticos, como aqueles associados a possibilidade da partícula circular o obstáculo várias vezes antes de atingir a tela. Porém, o modelo em que trabalhamos recorre a simplicidade, descartando essas contribuições, uma vez que, segundo Yabiki, o peso probabilístico desses eventos é relativamente desprezível. Para uma compreensão mais fina sobre este assunto, recomendamos as referências 49 55, onde os autores examinam as contribuições de caminhos não clássicos em sistemas de fendas dupla e tripla.

\section{Perfis gráficos da difração do modelo proposto}

Ao extrairmos o módulo ao quadrado da Eq. (19), obtemos as intensidades sobre a tela [56],

$$
\begin{aligned}
\mathcal{P}(x) & =\left|\Psi^{(+)}(x)\right|^{2}+\left|\Psi^{(-)}(x)\right|^{2} \\
& +\left(\Psi^{(-)^{*}} \Psi^{(+)}+\Psi^{(+)^{*}} \Psi^{(-)}\right)
\end{aligned}
$$

com a parcela de interferência dada por,

$$
\begin{aligned}
\mathcal{I}_{(+,-)}(x)= & \frac{\rho}{\lambda D \eta}\left[\left(\mathcal{C}\left(\zeta_{2}^{(-)}\right)-\mathcal{C}\left(\zeta_{1}^{(+)}\right)+\frac{1}{2}\right)+\left(\mathcal{S}\left(\zeta_{2}^{(-)}\right)-\mathcal{S}\left(\zeta_{1}^{(+)}\right)+\frac{1}{2}\right)\right. \\
& \left.-2\left(\mathcal{C}\left(\zeta_{2}^{(-)}\right) \mathcal{C}\left(\zeta_{1}^{(+)}\right)+\mathcal{S}\left(\zeta_{2}^{(-)}\right) \mathcal{C}\left(\zeta_{1}^{(+)}\right)\right)\right] .
\end{aligned}
$$

Com a soma das Eq. (12) e Eq. 22 na Eq. (21), somos capazes de traçar graficamente os padrões de difração dos elétrons frente ao obstáculo rígido e delgado, fixando valores para alguns parâmetros como $\eta$, por motivos já discutidos na seção 2 Fig. 5 e flutuando outros.

Quando discutidos os padrões de difração em [28 34], é suposto implicitamente que os objetos interpostos no 


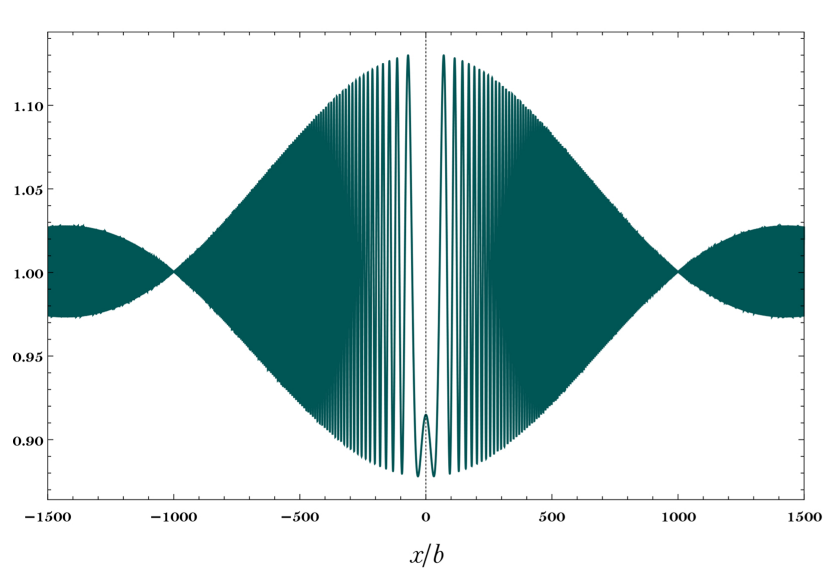

(a) $N_{F}(b)=0.001, \eta=2$.

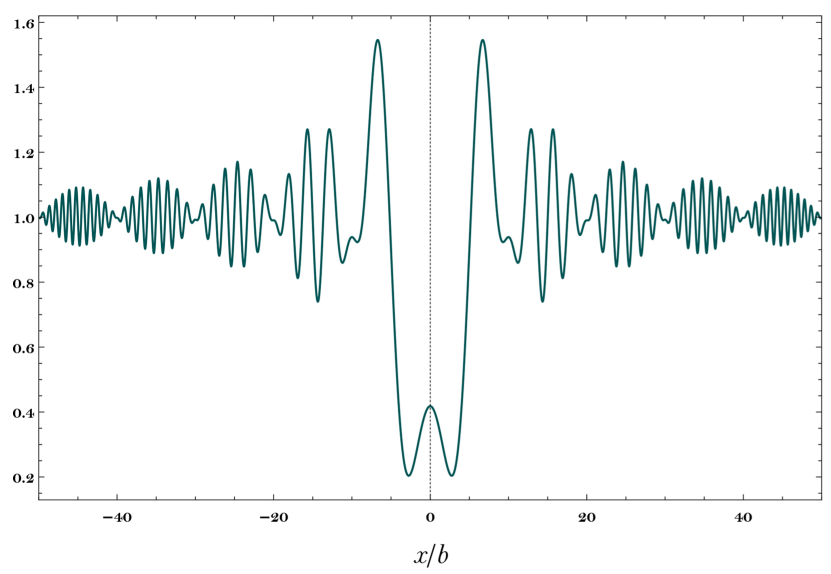

(c) $N_{F}(b)=0.1, \eta=2$.

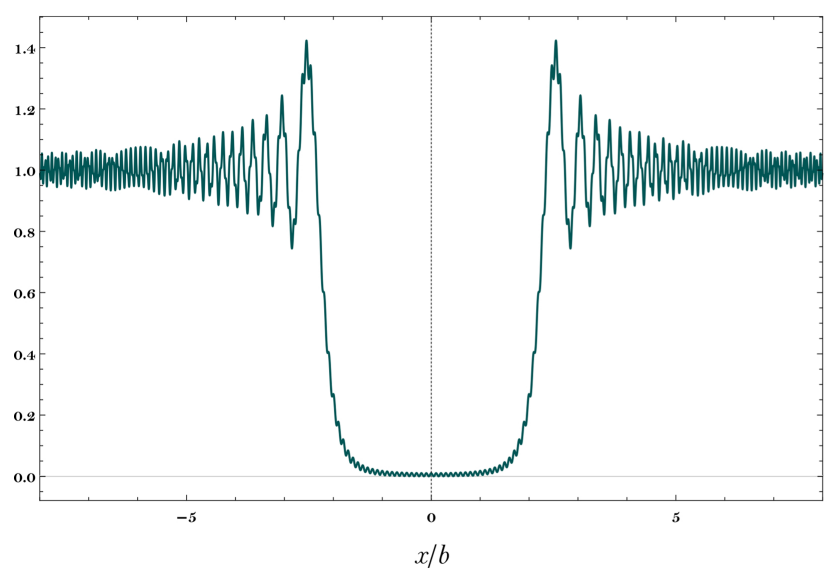

(e) $N_{F}(b)=10, \eta=2$.

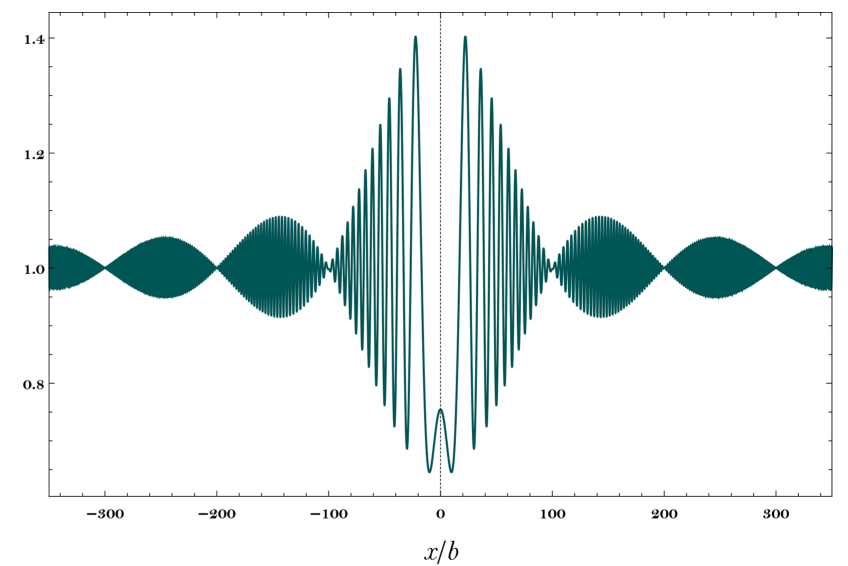

(b) $N_{F}(b)=0.01, \eta=2$.

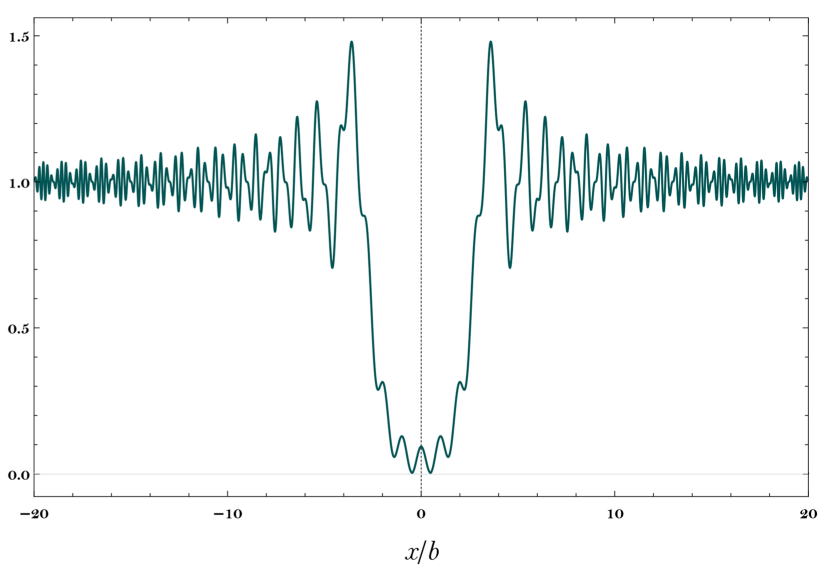

(d) $\quad N_{F}(b)=1, \eta=2$.

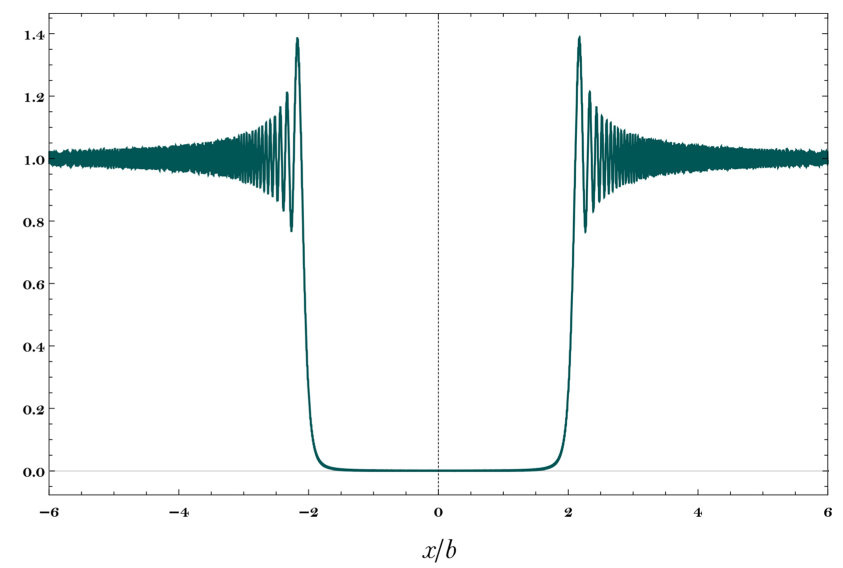

(f) $N_{F}(b)=100, \eta=2$.

Figura 6: Curvas de interferência geradas pela obstrução parcial da frente de onda de matéria por um fio delgado de tamanho variado. As abscissas são as distâncias dadas em unidades de $b$, com as ordenadas representando as intensidades.

caminho da onda desempenhavam um papel passivo, isto é, apenas interrompiam uma parte da frente de onda, sem adicionarem por si mesmos nenhuma nova onda. Com tal hipótese, os efeitos de difração observados são devidos exclusivamente à distorção do movimento ondulatório incidente. Como já mostrado na Fig. 4 , cada ponto da frente de onda que chega as semi fendas $( \pm)$ funciona como uma fonte secundária coerente. Dessa forma, a diferença mínima de caminho óptico entre elas (com relação a um ponto $\mathcal{X}$ sobre a tela), está associada as fontes secundárias mais próximas, situadas nas extremidades do obstáculo $\left(A^{\prime}, B^{\prime}\right)$, como pode ser visto na Fig. 7 Logo, se o comprimento de onda incidente é maior que $2 b$, será também maior que $\Delta r^{( \pm)}$. Considerando que as ondas, 


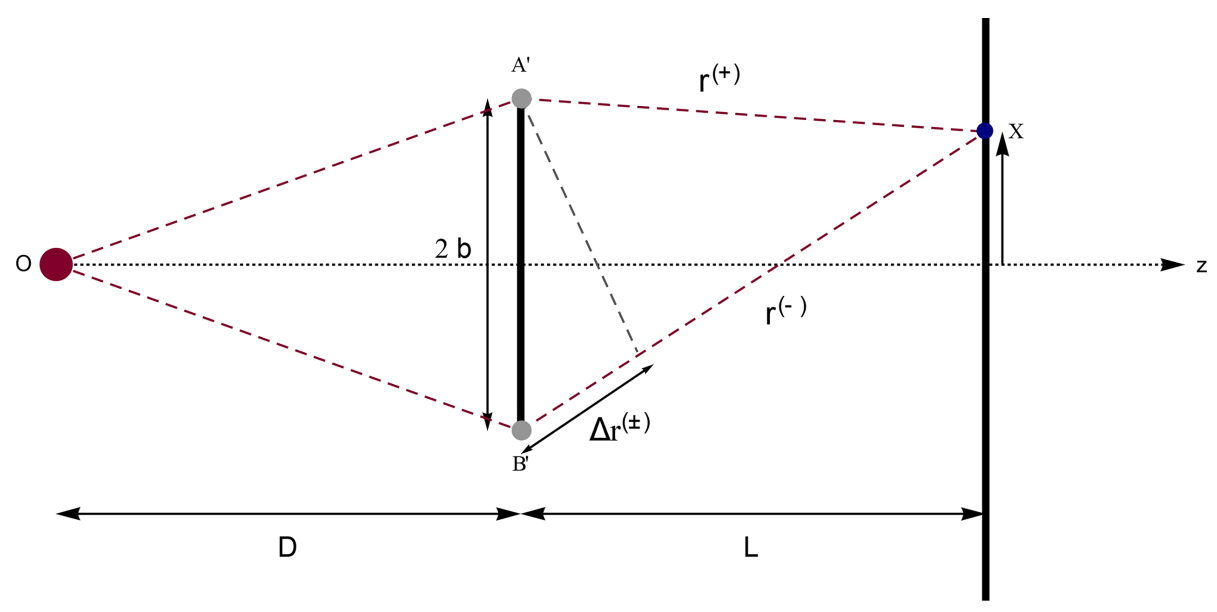

Figura 7: Esboço do aparato evidenciando a menor diferença de caminho óptico.

nas aberturas, estão inicialmente em fase, para além do anteparo elas vão todas interferir construtivamente em diferentes níveis, mas só interferem construtivamente porque a diferença de caminho óptico (ou diferença de fase) vai ser sempre bem menor que um comprimento de onda, qualquer que seja o ponto $\mathcal{X}$.

Percebemos claramente nos casos em que $N_{F}(b) \ll 1$ e $N_{F}(b) \leq 1$, que as intensidades, apesar de pequenas em alguns trechos, não são nulas. $\mathrm{O}$ que significa dizer também que essas regiões são iluminadas, pois a onda foi capaz de contornar todo o objeto e, portanto, penetrar por completo para além dos limites geométricos do obstáculo. Neste cenário, dizemos que ocorreu difração total da onda, prevalecendo a óptica ondulatória . Porém, a medida que $b$ aumenta o aspecto de onda estacionária (nas regiões abertas) se perde, ao mesmo tempo em que há a formação de uma região escura por detrás do objeto, denunciando que a probabilidade de encontrar elétrons ali é nula ou quase nula nas regiões próximas as bordas (ver Fig. 5 e Eq. (17)). Assim sendo, a zona de interferência destrutiva vai adquirindo os contornos do objeto denunciando o que chamamos de difração parcial da onda, com predominância da sombra geométrica (no domínio da óptica geométrica) na região imediatamente anterior ao obstáculo, a medida que nos aproximamos do limite $\frac{\lambda}{2 b} \rightarrow 0$.

No Cap.(33), seção (6) da Ref. [57], são comparados, qualitativamente, os padrões de difração (por luz) no regime de Fresnel de um disco opaco com o de uma abertura circular, dizendo que ambos são complementos um do outro. Isto se torna bem evidente ao compararmos a Fig. 6 com as Figs. $(5,6)$ da Ref. [58] e, especialmente, a Fig. (3-c) da Ref. 28. Este fato nos remete ao chamado princípio de Babinet, e para maiores detalhes sobre o assunto indicamos a Ref. [59], onde o autor aborda, teórica e experimentalmente, a difração de ondas gaussianas por um fio delgado sob o ponto de vista da aplicabilidade deste princípio.

\section{Os padrões de interferência e difração somados ao efeito de fase Aharonov-Bohm}

O trabalho que inaugurou as discussões sobre os efeitos locais dos potenciais eletromagnéticos surgiu em 1948, em uma atividade conjunta de Ehrenberg e Siday 60]. Aproximadamente uma decáda depois, em 1959, Y. Aharonov e D. Bohm 61] causaram grande surpresa e controvérsia ao demonstrarem que, no cenário da $\mathrm{MQ}$, os potenciais eletromagnéticos poderiam ter consequências observáveis sobre partículas carregadas como o elétron, ao atravessarem regiões em que os campos, elétrico e magnético, fossem nulos. Observaram assim que, na teoria quântica os potenciais eletromagnéticos possuiam um significado maior do que aquele que lhes eram atribuídos no domínio clássico da Física. Um tratamento com maior riqueza de detalhes sobre o assunto é dada por estes autores na Ref. 62, respondendo a uma série de questionamentos contrários as conclusões mencionadas em 61].

No eletromagnetismo clássico, os campos são quantidades mensuráveis e o conceito de força é fundamental, enquanto que seus potenciais são meras construções matemáticas sujeitas a transformações de calibre. Porém, ao tratar a dinâmica de sistemas quânticos, a equação de Schrödinger não envolve forças e campos e é necessariamente expressa em termos dos potenciais vetor e/ou escalar, o que os eleva ao status de quantidades fundamentais. Na Ref. 63 os autores introduzem de maneira simples, os conceitos de alguns eventos de natureza puramente quânticas, como o efeito Aharonov-Bohm (AB). Geralmente investigado em termos de teorias de calibre abelianas 60 62 com campos eletromagnéticos estacionários, M.Bright e D.Singleton 64 estudam o efeito $A B$ dependente do tempo para campos de gauge nãoabelianos 65. Eles fizeram uso de soluções dependentes do tempo bem conhecidos para as equações de campo de Yang-Mills e investigaram a mudança de fase $A B$.

A partir de 1960, com o trabalho de Chambers 66 
dá-se início a uma série de verificações experimentais do Efeito Aharonov-Bohm [67-69]. O caso em que é empregado um solenoide (suposto) infinito tem sido largamente explorado em estudos teóricos $31,70-72]$, fato que nos motiva também ao uso dessa ideia neste trabalho. No entanto, não deixamos de aludir sobre as dificuldades práticas em reproduzir um solenoide infinito, e por consequência disso, o difícil confinamento total do campo magnético em seu interior. A fim de evitar esse impasse, Tonomura et al. 73 75, em seus estudos experimentais, empregaram magnetos toroidais que encerraram por completo o campo magnético. Uma análise matemática rigorosa do efeito $A B$ com magnetos toroidais sob as condições experimentais de Tonomura et al. é feita na Ref. [76].

Em 1984, M. V. Berry 77] demonstra por meio da equação de Schrödinger que, se o sistema é preparado em um autoestado não degenerado do Hamiltoniano após uma evolução adiabática cíclica, ele retornará ao seu estado original acompanhado por um fator de fase formado por duas parcelas: uma contribuição dinâmica associada a energia do sistema e uma contribuição geométrica ligada ao caminho traçado no chamado espaço de parâmetros. Ainda neste trabalho, Berry verifica que o efeito $\mathrm{AB}$ pode ser interpretado como um exemplo de fase geométrica. Em sequência, vieram Y. Aharonov e J. Anandan [78, que propuseram uma forma mais geral para a fase geométrica de Berry, a fase Aharonov-Anandan, removendo a condição adiabática de qualquer evolução cíclica de um sistema quântico. J. Samuel e R. Bandari [79] deram outro passo importante na teoria aplicando a fase geométrica de Berry à evoluções não cíclicas e não unitárias de sistemas quânticos, baseados no trabalho de Pancharatman [80] sobre interferência de luz polarizada. $\mathrm{Na}$ Ref. [81], Anandan estende a teoria à fases geométricas não adiabáticas e não abelianas. Desse modo, é importante destacar que efeitos topológicos quânticos se manifestam nas mais diversas áreas da Física 82 88.

Como esboçado na Fig. 8 no interior do cilindro existe campo magnético $\mathbf{B}$, constante, paralelo ao eixo do solenoide e perpendicular ao plano da página. Em um primeiro momento, o feixe subdivide-se, recombinando-se num instante posterior em uma região sobre a tela. A função de onda recombinada, associada ao estado final das partículas carregadas, acumula uma fase quântica extra responsável pelo deslocamento das curvas de interferências observadas no padrão de distribuição das intensidades (Fig. 9), consequência da presença do potencial vetor.

A Lagrangiana do elétron pode ser dada em função do potencial vetor e da Lagrangiana na ausência do campo magnético, nomeada por $\mathcal{L}^{(0)}$, tal como na Ref. 89

$$
\mathcal{L}^{(0)}=\frac{1}{2} m \dot{\mathbf{x}}^{2} \longrightarrow \mathcal{L}^{(0)}+\frac{q}{c} \dot{\mathbf{x}} \cdot \mathbf{A}
$$

A correspondente mudança na ação, para qualquer segmento definido do caminho que conecta os pontos de coordenadas espaço-temporal $\left(\mathbf{x}_{n-1}, t_{n-1}\right)$ a $\left(\mathbf{x}_{n}, t_{n}\right)$ é dada por

$$
\mathcal{S}^{(0)}(n, n-1) \longrightarrow \mathcal{S}^{(0)}(n, n-1)+\frac{q}{c} \int_{t_{n-1}}^{t_{n}} \mathbf{A} \cdot \dot{\mathbf{x}} d t
$$

Porém, esta última integral pode ser reparametrizada, sendo escrita como

$$
\int_{t_{n-1}}^{t_{n}} \mathbf{A} \cdot\left(\frac{d \mathbf{x}}{d t}\right) d t=\int_{\mathbf{x}_{n-1}}^{\mathbf{x}_{n}} \mathbf{A} \cdot d \mathbf{x}
$$

em que $d \mathbf{x}$ é o elemento diferencial de linha por toda a extensão da trajetória, de maneira que, quando considerada a contribuição total entre $\mathcal{O}$ (onde se localiza a fonte emissora) a $\mathcal{X}^{\prime}$ (região de interferência), tem-se a seguinte alteração:

$$
\prod_{n} \exp \left[\frac{i \mathcal{S}^{(0)}(n, n-1)}{\hbar}\right] \rightarrow\left\{\prod_{n} \exp \left[\frac{i \mathcal{S}^{(0)}(n, n-1)}{\hbar}\right]\right\} \exp \left(\frac{i q}{\hbar c} \int_{\mathcal{O}}^{\mathcal{X}^{\prime}} \mathbf{A} \cdot d \mathbf{x}\right)
$$

Isso é verdadeiro para um caminho em particular, passando acima ou abaixo do aparato, obstáculo+solenóide. Portanto, ao considerar a contribuição de todos os caminhos possíveis temos o propagador da partícula ao deixar a fonte no ponto $\mathcal{O}$ e atingir a tela de interferência no ponto $\mathcal{X}^{\prime}$, representada por,

$$
\mathcal{K}\left(\mathcal{X}^{\prime} ; \mathcal{O}\right)=\int \exp \left[\frac{i \mathcal{S}^{(0)}\left(\mathcal{O} ; \mathcal{X}^{\prime}\right)}{\hbar}\right]\left\{\exp \left(\frac{i q}{\hbar c} \int_{\mathcal{O}}^{\mathcal{X}^{\prime}} \mathbf{A} \cdot d \mathbf{x}\right)\right\} \mathrm{D}[\mathbf{x}(t)] .
$$

A amplitude de transição ou função de onda total é dada pela soma das funções $\Psi_{A B}^{(+)}$e $\Psi_{A B}^{(-)}$associados, respectivamente, com as contribuições das trajetórias que passam por cima e por baixo do aparato, como representado na Fig. 8 assumindo a seguinte forma

$$
\Psi_{A B}(x)=\Psi_{A B}^{(+)}(x)+\Psi_{A B}^{(-)}(x) .
$$

Trajetórias que ao circularem o sistema se distanciaram muito das trajetórias clássicas, serão ignoradas, uma vez que pouco contribuem para a amplitude total [48]. Em cada parcela que compõe a função de onda na tela, há um 


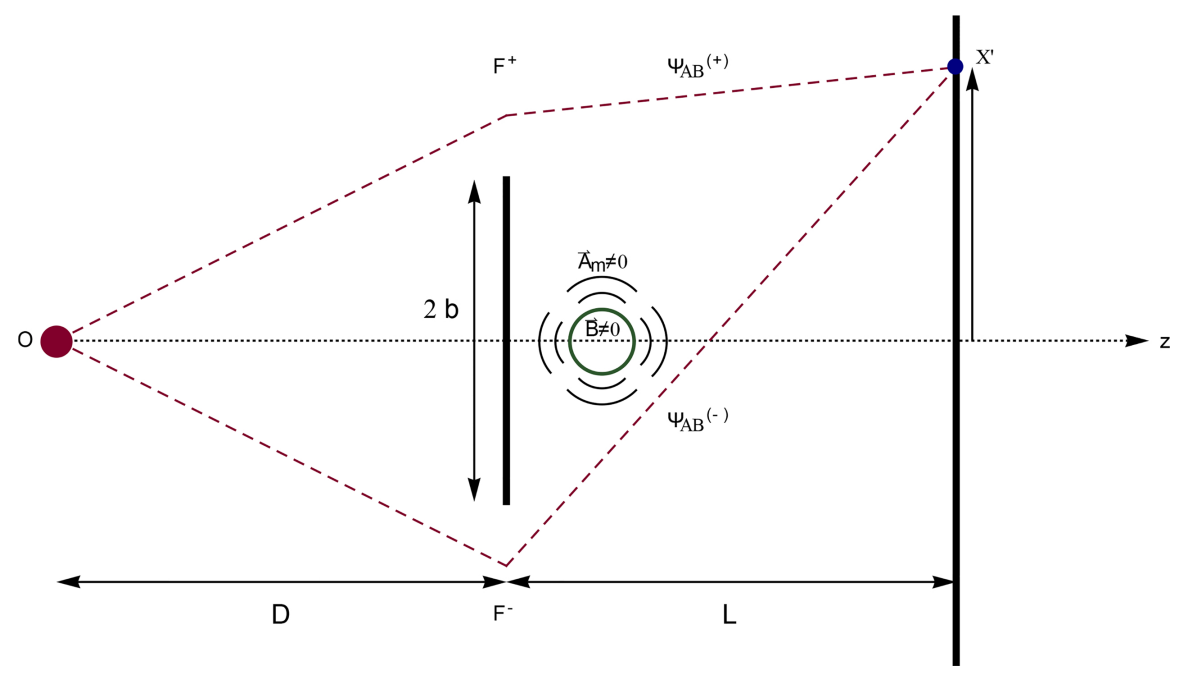

Figura 8: Efeito Aharonov-Bohm: o feixe de elétrons se divide e suas metades passam por ambos os lados de um obstáculo que oculta um solenoide suposto infinito de raio desprezível (para efeito representativo, seu esboço é exagerado), com fluxo de campo $\vec{B}$ em seu interior.

fator de fase do tipo $\left(\frac{q}{\hbar c} \int_{\mathcal{O}}^{\mathcal{X}^{\prime}} \mathbf{A} \cdot d \mathbf{x}\right)$ que expressa a dependência com o fluxo de campo magnético $\phi_{m}$ no interior do solenoide. Na região em que a carga se encontra o campo, dado por $\mathbf{B}=\nabla \times \mathbf{A}$, é nulo e as integrais de linha de $\mathbf{A}$ acima e abaixo do solenoide dependem apenas dos pontos terminais $\mathcal{O}$ e $\mathcal{X}^{\prime}$ e não de trajetórias específicas quando formado um laço por caminhos diferentes. Assim, as exponenciais que contém A são independentes das trajetórias sob as quais se está integrando. Com isso, escrevemos as funções de onda provenientes de cada ramo como,

$$
\begin{aligned}
\Psi_{A B}^{( \pm)}(x) & =\exp \left(\frac{i q}{\hbar c} \int_{\mathcal{O}}^{\mathcal{X}^{\prime}} \mathbf{A} \cdot d \mathbf{x}\right)^{( \pm)} \Psi^{( \pm)}(x), \\
& =e^{i \Omega^{( \pm)}} \Psi^{( \pm)}(x) .
\end{aligned}
$$

As distribuições eletrônicas sobre a tela de interferência são dadas pelo quadrado do módulo da amplitude de transição total (Eq. 28)

$$
\begin{aligned}
\mathcal{P}_{A B}(x)= & \left|\Psi^{(+)}(x)\right|^{2}+\left|\Psi^{(-)}(x)\right|^{2}+\left(\Psi^{(-)^{*}} \Psi^{(+)}+\Psi^{(+)^{*}} \Psi^{(-)}\right) \cos \left(\Phi_{A B}\right) \\
& +i\left(\Psi^{(-)^{*}} \Psi^{(+)}-\Psi^{(+)^{*}} \Psi^{(-)}\right) \sin \left(\Phi_{A B}\right) .
\end{aligned}
$$

Em relação a Eq. 21], percebemos que existem duas parcelas de interferência ponderadas por funções trigonométricas que guardam informações acerca do fluxo. A primeira delas já é conhecida (ver Eq. (22)), com a segunda parcela sendo representada por

$$
\begin{aligned}
\mathcal{H}_{(+,-)}(x)= & i\left(\Psi^{(-)^{*}} \Psi^{(+)}-\Psi^{(+)^{*}} \Psi^{(-)}\right) \\
= & \frac{\rho}{\lambda D \eta}\left[\left(\mathcal{S}\left(\zeta_{2}^{(-)}\right)-\mathcal{C}\left(\zeta_{2}^{(-)}\right)\right)+\left(\mathcal{S}\left(\zeta_{1}^{(+)}\right)-\mathcal{C}\left(\zeta_{1}^{(+)}\right)\right)\right. \\
& \left.-2\left(\mathcal{C}\left(\zeta_{1}^{(+)}\right) \mathcal{S}\left(\zeta_{2}^{(-)}\right)-\mathcal{S}\left(\zeta_{1}^{(+)}\right) \mathcal{C}\left(\zeta_{2}^{(-)}\right)\right)\right] .
\end{aligned}
$$

Essas funções surgem da combinação das exponenciais presentes na Eq. 29], gerando uma integral de linha fechada do potencial vetor em torno do obstáculo + sole- noide (feita no sentido anti-horário ou trigonométrico de giro), a qual pode ser identificada como a diferença de fase entre as contribuições dos caminhos acima e abaixo 
desse sistema chamado de parâmetro de fluxo

$$
\begin{aligned}
\Phi_{A B} & =\frac{q}{\hbar c}\left(\int_{\mathcal{O}}^{\mathcal{X}^{\prime}} \mathbf{A} \cdot d \mathbf{x}+\int_{\mathcal{X}^{\prime}}^{\mathcal{O}} \mathbf{A} \cdot d \mathbf{x}\right) \\
& =\left(\frac{q}{\hbar c}\right) \oint_{\mathcal{C}} \mathbf{A} \cdot d \mathbf{x} \\
& =\frac{q}{\hbar c} \phi_{m} .
\end{aligned}
$$

Na Ref. 31] D. Shapiro e W. Henneberger introduzem o efeito de fase $\mathrm{AB}$ (padrão) no experimento de fenda dupla (com função de fenda definida por uma função degrau apesar de não coloca-la explicitamente, em uma clara melhoria do modelo abordado em [29], onde foi usado funções gaussianas para representar as regiões de fenda) com elétrons na presença de um whisker 11 Seus resultados foram obtidos via método numérico de Romberg na avaliação das funções de Fresnel. Na Ref. [34], K. Bradonjić e J. D. Swain vão além, assumindo que o solenoide está em uma superposição de dois estados macroscópicos correspondentes a correntes opostas de igual magnitude e que por consequência disso, geram campos magnéticos superpostos (com orientações positiva (up) ou negativa (down)) em seu interior. Salientam que a informação sobre o estado do fluxo superposto é aferida do padrão de interferência sem colapsa-lo (a princípio, em um dos estados up ou down), uma vez que este é formado pelas contribuições coletivas das dispersões eletrônicas repetidas em grande número. Esclarecem ainda, que seus esforços foram motivados pelas realizações experimentais das referências 90 91 . 91 onde os respectivos autores tratam sobre o assunto empregando anéis supercondutores. Além de sugerirem aplicações na computação quântica para determinar informações sobre os estados de qubits 92 95. armazenados como superposições de fluxos magnéticos. Considerações relacionadas ao efeito $\mathrm{AB}$ e deslocamentos no padrão de interferência (por fenda dupla) via equação de Schrödinger, também podem ser encontrados em [96].

A partir da Eq. (30), traçamos diferentes perfis gráficos de difração, com efeito de fase $\mathrm{AB}$, preservando a sequência dada na Fig. 6. com o parâmetro de fluxo fixo, para efeito de comparação.

Nitidamente, a assimetria causada pela fase $\mathrm{AB}$ é mais proeminente nos casos em que $N_{F}(b) \ll 1$, isto é, nos regimes de difração total, tornando-se menos perceptível a medida que $N_{F}(b)$ cresce e se afasta da unidade. Como pode ser visto na Fig. 7 se o comprimento de onda incidente é menor que $2 b$, será também menor que $\Delta r^{( \pm)}, \mathrm{com}$ as parcelas 22 e 31 assumindo valores significativos. Se as ondas estão em fase nas aberturas, na zona imediatamente atrás do obstáculo elas irão todas interferir destrutivamente (já que diferença de caminho óptico (ou diferença de fase) vai ser sempre bem maior que um comprimento de onda), salvo as regiões próximas as suas extremidades, da ordem de poucos comprimentos de onda, onde ainda são parcialmente iluminadas (Fig. 94d), com pequenos desvios AB (Fig. 9 e) ou desvios AB inexistentes (Fig. 9-f). Nesses casos, a Eq. (30) se comporta como a Eq. (21) para $N_{F}(b)$ extremamente grande.

\section{Considerações finais e Conclusões}

Motivados por um argumento dado por D.H.Kobe em [29], abordamos o problema de difração de ondas de matéria (elétrons) por um obstáculo rígido, não interagente e delgado via integrais de caminho de Feynman, alternativamente a teoria escalar da difração via equação de Schrödinger e o teorema integral de Kirchhoff. A definição da função (5), nos permitiu, de forma natural, eliminar as paredes que se estendiam para as regiões além das fendas na Ref. [31], restando apenas uma das paredes centrada, que funcionou como o fio delgado interposto no caminho da onda de matéria. Recuperamos os perfis gráficos (Fig. 6), com mais detalhes que os apresentados na Fig. 6 da Ref. [97], onde o autor apresentou um método novo, para a época, de diagnóstico para medidas de estruturas de alta densidade em pequenas escalas com plasmas quentes. Tudo isso a partir de expressões analíticas, com base na relação entre o comprimento de onda e o tamanho característico do obstáculo via número de Fresnel $N_{F}(b)$, deixando claro o predomínio, em cada caso, da óptica geométrica e física na região imediatamente anterior a este obstáculo.

Com respeito ao conteúdo pertinente a teoria tratada aqui, subdividimos a literatura tradicionalmente adotada em cursos de graduação de ciências exatas (Física, Engenharia e Matemática), acerca de sua complexidade, em dois grupos: (I) ministradas ao ciclo básico [47, 57, 98]; (II) destinadas às disciplinas mais avançadas, como o Eletromagnetismo 99], Óptica 100] e Mecânica Quântica [89]. Em ambos os grupos os autores apresentam, de uma maneira ou outra, o fenômeno da difração e interferência de luz (e elétrons) por fendas simples e dupla, incluindo a difração por uma borda ou parede semi infinita, recuperado na seção 2 - Fig. 5 porém, nada dizem sobre o comportamento deste último mediante variação do parâmetro $\eta$, isto é, não há qualquer comentário sobre o efeito produzido sobre este padrão mediante variação das distâncias relativas entre os eixos da borda e da tela de difração, evitando assim, qualquer conclusão equivocada que o estudante venha a ter. O mesmo pode ser dito em relação ao esboço dos gráficos de difração por um obstáculo delgado (Fig. 6), comparativamente a $\lambda$, o que tornaria visualmente claro a eles, que fenômenos ondulatórios e de interferência não se limitam apenas a fenda simples ou dupla, reiterando assim, o argumento dado na introdução do trabalho de Daniel et al. 23. É válido dizer também, que a representação das integrais de caminho é perfeitamente cabível ao problema de difração por fenda retangular (ou genérica) feito em 23], bastando para isso que o leitor reescreva adequadamente a função

${ }^{1}$ Em inglês significa cerda ou fio de bigode; no presente contexto toma-se por um filamento delgado de ferro magnetizado. 


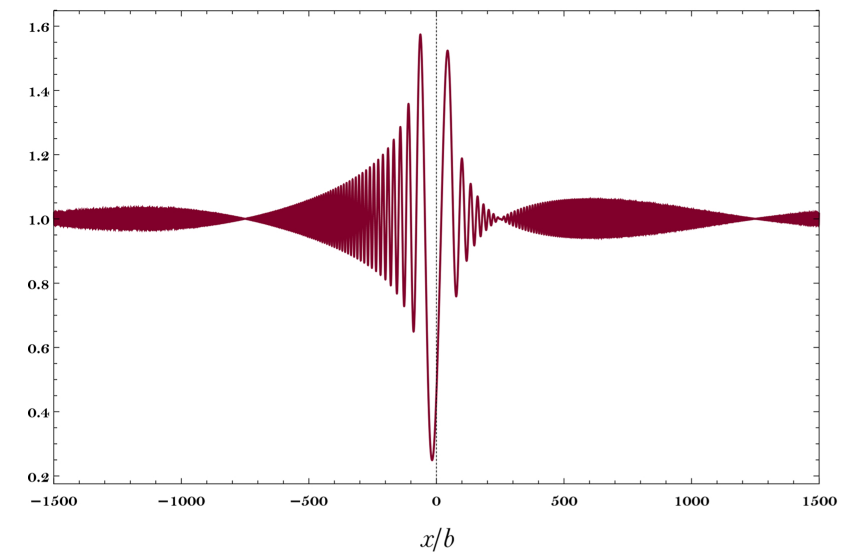

(a) $N_{F}(b)=0.001, \eta=2, \Phi_{A B}=\pi / 2$.

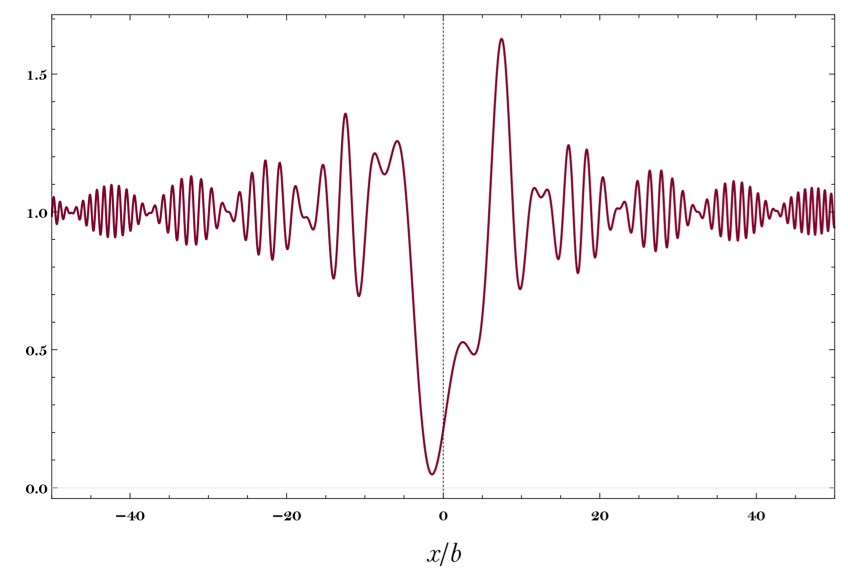

(c) $N_{F}(b)=0.1, \eta=2, \Phi_{A B}=\pi / 2$.

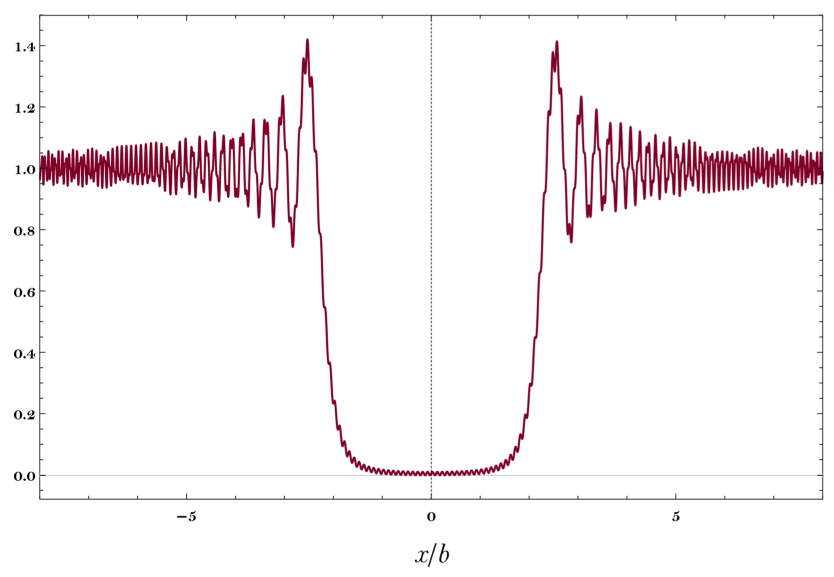

(e) $N_{F}(b)=10, \eta=2, \Phi_{A B}=\pi / 2$.

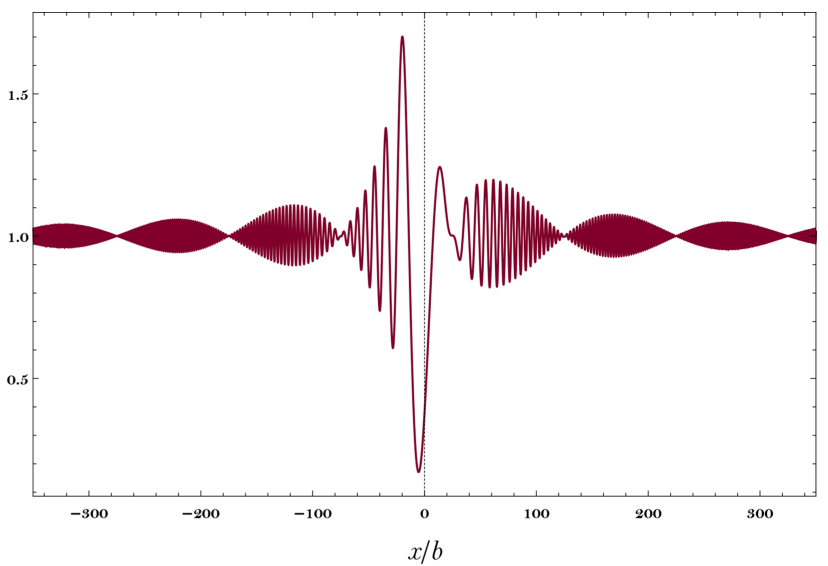

(b) $N_{F}(b)=0.01, \eta=2, \Phi_{A B}=\pi / 2$.

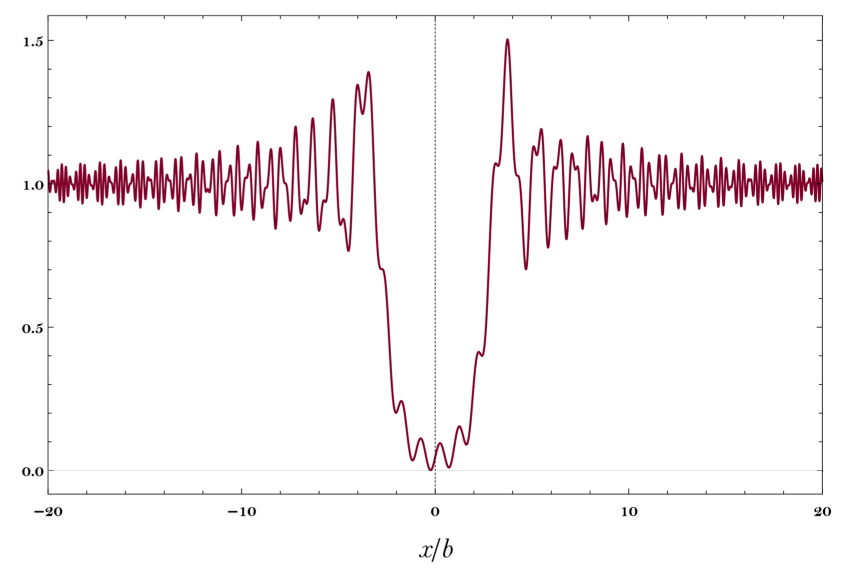

(d) $N_{F}(b)=1, \eta=2, \Phi_{A B}=\pi / 2$.

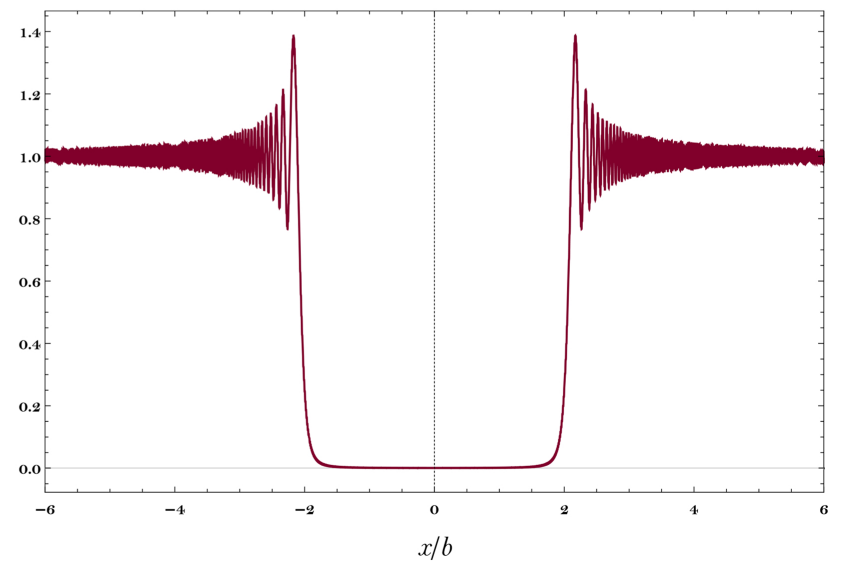

(f) $N_{F}(b)=100, \eta=2, \Phi_{A B}=\pi / 2$.

Figura 9: Curvas de interferência e difração geradas pela obstrução parcial da frente de onda de matéria com efeito de fase $A B$. As abscissas são as distâncias dadas em unidades de $b$, com as ordenadas representando as intensidades.

dada pela Eq. (5) e construa corretamente os propagadores associados a cada etapa do movimento (como na Eq. (3) e na Eq. (4)).

Ao longo do tempo, uma série de trabalhos envolvendo interferometria em sistemas de fenda dupla empregavam, figurativamente, um solenoide na região entre fendas (atrás de um obstáculo) $29,31-34]$. O mesmo ocorre na seção 4 desse trabalho, ao posicionarmos um solenoide na região imediatamente anterior ao fio, de modo que seu raio sempre obedecerá a condição $r_{\text {sol }} \ll 2 b$, não nos causando assim, nenhum prejuízo conceitual. Isto nos permitiu, a partir da Eq. 30), extrair os perfis gráficos de interferência somados aos efeitos de fase AB com diferentes níveis de assimetria, conforme variava-se o com- 
primento do fio (ver Fig. 9p, inéditos até então, que por sua vez, podem ser usados como recurso complementar no estudo do Cap. (2), seção (2.6), da Ref. [89] (grupo (II)). No entanto, ficou claro que a partir de $\bar{N}_{F}(b)>1$ tornase difícil a tarefa de distinguir as assimetrias do efeito AB (devido a formação de sombra e a predominância das parcelas associadas as regiões abertas $( \pm)$ na Eq. (30), e portanto, neste regime o modelo deixa de ser o mais indicado para esse tipo de análise. Algo semelhante ocorrerá em relação aos padrões de difração e interferência por fenda dupla, discutidos na Ref. [28], nos regimes de Fresnel e Fraunhofer com fases separadas quando adicionados os efeitos de fase AB. Nestes casos, em particular no primeiro regime (admitindo que o tamanho das fendas seja muito maior que $\lambda$, com a parede entre elas muito maior que as próprias fendas), surgirá uma região de sombra (semelhante a Fig. 9ff) entre as porções iluminadas, com quase imperceptíveis assimetrias nos limites geométricos das fendas em virtude da insignificância dos valores assumidos pelas parcelas de interferências. Logo, assim como na Fig. 9 f, este regime é igualmente não recomendável às observações do efeito $\mathrm{AB}$, e sobre isto, curiosamente, nada foi dito nas Refs. 29 31. Nesta perspectiva, o leitor poderá se certificar também que ao aplicar o efeito $A B$ superposto descrito por K. Bradonjić e J. D. Swain ao modelo proposto na Fig. 8, e recorrendo ao número de Fresnel $N_{F}(b)$, será capaz de construir graficamente as configurações das intensidades em diferentes regimes e combinações de fluxo, semelhantes as Fig. 3 e Fig. 4 da Ref. 34] para $N_{F}(b) \gg 1$, porém, bem diferentes quando $N_{F}(b) \ll 1$.

Nesses últimos anos, com o avanço crescente da tecnologia, trabalhos teóricos e experimentais que tratam mecanismos em escalas micro e nanométricas são cada vez mais possíveis $101-103$. Em 104 os autores lidam com experimentos envolvendo a manipulação e transmissão de luz por fendas de mesma ordem ou de ordem menor que o comprimento de onda do feixe incidente. Em 105], são investigadas o comportamento de ondas plasmônicas superficiais por fenda dupla, e como elas podem ser afetadas pela variação do tamanho da parede entre essas fendas (isso se torna nítido em nossas imagens, Fig. 6] e Fig. 9, para efeitos decorrentes da difração por essa parede isoladamente). Contudo, em um dado momento, a confecção de nano fendas ou mecanismos similares torna-se difícil ou impraticável para fins experimentais, e tal como em [106], dadas certas circunstâncias, a largura das fendas podem contribuir muito pouco nas medidas aferidas, corroborando para adoção de modelos com uma simples barreira como obstáculo.

Por fim, esperamos ter esclarecido de maneira contextualizada um pouco mais sobre os aspectos fundamentais da Física, no que diz respeito a óptica geométrica e física, apresentadas nas muitas referências citadas ao longo deste trabalho.

\section{Agradecimentos}

C.C. Favarato agradece o apoio financeiro da CAPES.

\section{Referências}

[1] L. de Broglie, Nature 112, 540 (1923).

[2] C.J. Calbick, The Physics Teacher 1, 63 (1963).

[3] R.B. Leighton e M.L.M. Sands, The Feynman Lectures on Physics: Quantum mechanics (Dover Publications, New York, 2012).

[4] R.P. Feynman e A.R. Hibbs, Quantum mechanics and path integrals: Emended edition (Dover Publications, New York, 2012).

[5] R. Bach, D. Pope, S.H. Liou e H. Batelaan, New Journal of Physics 15, 033018 (2013).

[6] C. Jönsson, Zeitschrift für Physik 161, 454 (1961).

[7] C. Jönsson, American Journal of Physics 42, 4 (1974).

[8] O. Donati, G.P. Missiroli e G. Pozzi, American Journal of Physics 41, 639 (1973).

[9] P.G. Merli, G.F. Missiroli e G. Pozzi, American Journal of Physics 44, 306 (1976).

[10] A. Tonomura, J. Endo, T. Matsuda, T. Kawasaki e H. Ezawa, American Journal of Physics 57, 117 (1989).

[11] R. Rosa, Physics in perspective 14, 178 (2012).

[12] B. Barwick, G. Gronniger, L. Yuan, S.H. Liou e H. Batelaan, Journal of Applied Physics 100, 074322 (2006).

[13] S. Frabboni, C. Frigeri, G.C. Gazzadi e G. Pozzi, American Journal of Physics 79, 615 (2011).

[14] A. Zeilinger, R. Gähler, C.G. Shull, W. Treimer e W. Mampe, Reviews of Modern Physics 60, 1067 (1988).

[15] F. Shimizu, K. Shimizu e H. Takuma, Physical Review A 46, R17 (1992).

[16] O. Carnal e J. Mlynek, Physical Review Letters 66, 2689 (1991)

[17] M. Arndt, O. Nairz, J.V. Andreae, C. Keller, G. Van der Zouw e A. Zeilinger, Nature 401, 680 (1999).

[18] O. Nairz, M. Arndt e A. Zeilinger, Journal of Modern Optics 47, 2811 (2000).

[19] O. Nairz, M. Arndt e A. Zeilinger, American Journal of Physics 71, 319 (2003).

[20] M. Gondran e A. Gondran, American Journal of Physics 73, 507 (2005).

[21] X.Y. Wu, B.J. Zhang, Z. Hua, X.J. Liu, Y.H. Wu, H.L. Tang e J.W. Li, International Journal of Theoretical Physics 49, 2191 (2010).

[22] A.S. Sanz, F. Borondo e M.J. Bastiaans, Physical Review A 71, 042103 (2005).

[23] D.M. Reis, E.M. Santos e A.V.A. Neto, Revista Brasileira de Ensino de Física 37, 2312 (2015).

[24] D. Lincoln, The Physics Teacher 54, 203 (2016).

[25] A. Velentzas, The Physics Teacher 52, 493 (2014).

[26] D. F. Styer, M. S. Balkin, K. M. Becker, M. R. Burns, C. E. Dudley, S. T. Forth, J. S. Gaumer, M. A. Kramer, D. C. Oertel, L. H. Park, M. T. Rinkoski, C. T. Smith e T.D.Wotherspoon, American Journal of Physics 70, 288 (2002).

[27] P.D. Mannheim, American Journal of Physics 51, 328 (1983).

[28] M. Beau, European Journal of Physics 33, 1023 (2012).

[29] D.H. Kobe, Annals of Physics 123, 381 (1979). 
[30] O.A. Barut e S. Basri, American Journal of Physics 60, 896 (1992).

[31] D. Shapiro e W.C. Henneberger, Journal of Physics A: Mathematical and General 22, 3605 (1989).

[32] D.H. Kobe, V.C. Aguilera-Navarro e R.M. Ricotta, Physical Review A 45, 6192 (1992).

[33] V.C. Aguilera-Navarro e R.M. Quick, Physical Review A 50, 2885 (1994).

[34] K. Bradonjić e J.D. Swain, Quantum Information Processing 13, 323 (2014).

[35] R.P. Feynman, Reviews of Modern Physics 20, 367 (1948).

[36] P.A.M. Dirac, Proceedings of the Royal Society of London. Series A 133, 60 (1931).

[37] J.M.F. Bassalo e M.S.D. Cattani, Elementos de Física matemática, v.3 Equações integrais e Integrais de trajetória não relativísticas (Editora Livraria da Física, São Paulo, 2012) $1^{a}$ ed.

[38] A.V. Wijngaarden e W.L. Scheen, Table of Fresnel Integrals, (Verhandl. Konink. Ned. Akad. Wetenschapen., Amsterdam, 1949)

[39] R.J. Hangelbroek, Journal of Engineering Mathematics 1, 37 (1967).

[40] R. Bulirsch, Numerische Mathematik 9, 380 (1967).

[41] D.S. Meek e D.J. Walton, Journal of Computational and Applied Mathematics 25, 69 (1989).

[42] D.J. Walton e D.S. Meek, Computers \& Graphics 29, 353 (2005).

[43] D.J. Walton e D.S. Meek, Journal of Computational and Applied Mathematics 223, 86 (2009).

[44] L. Blanch, E. Checa e J. Marín, Modelling in Science Education and Learning 6, 105 (2013).

[45] J.L. Bastardo, S.A. Ibrahim, P.F. de Córdoba, J.F.U. Schölzel e Y.L. Ratis, Applied Mathematics Letters 18, 23 (2005).

[46] Y.Z. Umul, Optics Express 13, 8469 (2005).

[47] M. Alonso e E.J. Finn, Física um curso universitário, Campos e ondas (Editora Edgar Blucher, São Paulo 2015) v. 2, $2^{a}$ ed.

[48] H. Yabuki, International Journal of Theoretical Physics 25, 159 (1986).

[49] R.D. Sorkin, Modern Physics Letters A 09, 3119 (1994).

[50] U. Sinha, C. Couteau, T. Jennewein, R. Laflamme e G. Weihs, Science 329, 418 (2010).

[51] H. de Raedt, K. Michielsen e K. Hess, Physical Review A 85, 012101 (2012).

[52] R. Sawant, J. Samuel, A. Sinha, S. Sinha e U. Sinha, Physical Review Letters 113, 120406 (2014).

[53] A. Sinha, A.H. Vijay e U. Sinha, Scientific Reports 5, 10304 (2015).

[54] O.S. Magana-Loaiza, I. de Leon, M. Mirhosseini, R. Fickler, A. Safari, U. Mick, B. McIntyre, P. Banzer, B. Rodenburg, G. Leuchs e et al., Nature Communications 7, 13987 (2016).

[55] J.Q. Quach, Physical Review A 95, 042129 (2017).

[56] M. Born, Zeitschrift für Physik 38, 803 (1926).

[57] P.A. Tipler e G. Mosca, Física para cientistas e engenheiros, Eletricidade e magnetismo, Óptica (Editora LTC, Rio de Janeiro 2006), v. 2, $2^{a}$ ed.

[58] R.E. Haskell, American Journal of Physics 38, 1039 (1970).

[59] S. Ganci, American Journal of Physics 73, 83 (2005).
[60] W. Ehrenberg e R.E. Siday, Proceedings of the Physical Society. Section B 62, 8 (1949).

[61] Y. Aharonov e D. Bohm, Physical Review 115, 485 (1959).

[62] Y. Aharonov e D. Bohm, Physical Review 123, 1511 (1961).

[63] G.M. Amaral, D.Q. Aruquipa, L.F.M. Camacho, L.F.C Faria, S.I.C. Guzmán, D.T. Maimone, M. Mendes e M.A.P. Lima, Revista Brasileira de Ensino de Física 38, e3309 (2016).

[64] M. Bright e D. Singleton, Physical Review D 91, 085010 (2015).

[65] T.T. Wu e C.N. Yang, Physical Review D 12, 3845 (1975).

[66] R.G. Chambers, Physical Review Letters 5, 3 (1960).

[67] S. Olariu e I.I. Popescu, Reviews of Modern Physics 57, 339 (1985).

[68] B.E. Allman, A. Cimmino, A.G. Klein, G.I. Opat, H. Kaiser e S.A. Werner, Physical Review Letters 68, 2409 (1992).

[69] B.E. Allman, A. Cimmino, A.G. Klein, G.I. Opat, H. Kaiser e S.A. Werner, Physical Review Letters 70, 250 (1993).

[70] C.C. Gerry e V.A. Singh, Physical Review D 20, 2550 (1979).

[71] M.V. Berry, European Journal of Physics 1, 240 (1980).

[72] X. Zhu e W.C. Henneberger, Journal of Physics A: Mathematical and General 23, 3983 (1990).

[73] N. Osakabe, T. Matsuda, T. Kawasaki, J. Endo, A. Tonomura, S. Yano e H. Yamada, Physical Review A 34, 815 (1986).

[74] A. Tonomura, T. Matsuda, R. Suzuki, A. Fukuhara, N. Osakabe, H. Umezaki, J. Endo, K. Shinagawa, Y. Sugita e H. Fujiwara, Physical Review Letters 48, 1443 (1982).

[75] A. Tonomura, N. Osakabe, T. Matsuda, T. Kawasaki, J. Endo, S. Yano e H. Yamada, Physical Review Letters 56, 792 (1986).

[76] M. Ballesteros e R. Weder, Journal of Mathematical Physics 50, 122108 (2009).

[77] M.V. Berry, Proceedings of the Royal Society of London A: Mathematical, Physical and Engineering Sciences 392, 45 (1984).

[78] Y. Aharonov e J. Anandan, Physical Review Letters 58, 1593 (1987).

[79] J. Samuel e R. Bhandari, Physical Review Letters 60, 2339 (1988).

[80] S. Pancharatnam, Proceedings of the Indian Academy of Sciences - Section A 44, 247 (1956).

[81] J. Anandan, Physics Letters A 133, 171 (1988).

[82] R.B. Laughlin, Physical Review Letters 50, 1395 (1983).

[83] R.A. Webb, S. Washburn, C.P. Umbach e R.B. Laibowitz, Physical Review Letters 54, 2696 (1985).

[84] M. Bayer, M. Korkusinski, P. Hawrylak, T. Gutbrod, M. Michel e A. Forchel, Physical Review Letters 90, 186801 (2003).

[85] S. Russo, J.B. Oostinga, D. Wehenkel, H.B. Heersche, S.S. Sobhani, L.M.K. Vandersypen e A.F. Morpurgo, Physical Review B 77, 085413 (2008).

[86] M. Huefner, F. Molitor, A. Jacobsen, A. Pioda, C. Stampfer, K. Ensslin e T. Ihn, New Journal of Physics 12, 043054 (2010). 
[87] P. Recher, B. Trauzettel, A. Rycerz, Y.M. Blanter, C.W.J. Beenakker e A.F. Morpurgo, Physical Review B 76, 235404 (2007).

[88] L.L. Li, D. Moldovan, P. Vasilopoulos e F.M. Peeters, Physical Review B 95, 205426 (2017).

[89] J.J. Sakurai e J. Napolitano, Mecânica Quântica Moderna (Editora Bookman, Porto Alegre, 2013), $2^{a}$ ed.

[90] J.R. Friedman, V. Patel, W. Chen, S.K. Tolpygo e J.E. Lukens, Nature 406, 43 (2000).

[91] C.H. Van der Wal, A.C.J. ter Haar, F.K. Wilhelm, R.N. Schouten, C.J.P.M. Harmans, T.P. Orlando, S. Lloyd e J.E. Mooij, Science 290, 773 (2000).

[92] J.E. Mooij, T.P. Orlando, L. Levitov, L. Tian, C.H. Van der Wal e S. Lloyd, Science 285, 1036 (1999).

[93] T.P. Orlando, S. Lloyd, L.S. Levitov, K.K. Berggren, M.J. Feldman, M.F. Bocko, J.E. Mooij, C.J.P. Harmans e C.H. Van der Wal, Physica C: Superconductivity 372, 194 (2002).

[94] T.P. Orlando, L. Tian, D.S. Crankshaw, S. Lloyd, C.H. Van der Wal, J.E. Mooij, e F.Wilhelm, Physica C: Superconductivity 368, 294 (2002).

[95] T.P. Orlando, J.E. Mooij, L. Tian, C.H. Van der Wal, L.S. Levitov, S. Lloyd e J.J. Mazo, Physical Review B 60, 15398 (1999).

[96] T.H. Boyer, Physical Review D 8, 1679 (1973).
[97] P. Choi, C. Dumitrescu, E. Wyndham, M. Favre e H. Chuaqui, Review of Scientific Instruments 73, 2276 (2002).

[98] H.M. Nussenzveig, Curso de Física Básica, v. 4 Ótica, Relatividade e Física Quântica (Editora Edgar Blucher, São Paulo, 1998), $1^{a}$ ed.

[99] K.D. Machado, Eletromagnetismo, v. 3 Ótica, Relatividade e Física Quântica (Editora Toda Palavra, Ponta Grossa, 2013), $1^{a}$ ed.

[100] M. Born e E. Wolf, Principles of Optics - Eletromagnetic Theory of Propagation, Interference and Difraction of Light (Cambridge University Press, Cambridge, 1999), 7 th ed.

[101] H. Shi, C. Wang, C. Du, X. Luo, X. Dong e H. Gao, Optics Express 13, 6815 (2005).

[102] H. Yanagisawa, M. Ciappina, C. Hafner, J. Schötz, J. Osterwalder e M.F. Kling, Scientific Reports 7, 12661 (2017).

[103] Q. Chen, Y. Wang, T. Deng e Z. Liu, Nanotechnology 29, 085301 (2018)

[104] T.W. Ebbesen, H.J. Lezec, H.F. Ghaemi, T. Thio e P.A. Wolff, Nature 391, 667 (1998).

[105] M. Verma, S. Joshi, N.S. Bisht, H.C. Kandpal, P. Senthilkumaran e J. Joseph, Journal of the Optical Society of America A 29, 195 (2012).

[106] S. You, C. Kuang e B. Zhang, Scientific Reports 6, 33764 (2016). 